United States Department of Energy

Grant No. DE-FG05-88ER40446

\title{
MEDIUM ENERGY MEASUREMENTS OF N-N PARAMETERS
}

\section{PROGRESS REPORT}

\section{For}

the three year period

January 1, 1988 - December 31, 1990

\author{
PRINCIPAL INVESTIGATOR \\ Peter J. Riley \\ Department of Physics \\ University of Texas at Austin \\ Austin, Texas, 78712
}

October 1, 1990

\section{DISCLAIMER}

This report was prepared as an account of work sponsored by an agency of the United States Government. Neither the United Staies Government nor any agency thereof, nor any of their employees, makes any warranty, express or implied, or assumes any legal liability or responsibility for the accuracy, completeness, or usefulness of any information, apparatus, product, or process disclosed, or represents that its use would not infringe privately owned rights. Reference herein to any specific commercial product, process, or service by trade name, trademark, manufacturer, or otherwise does not necessarily constitute or imply its endorsement, recornmendation, or favoring by the United States (iovernment of any agency thereof. The views and opinions of authors expressed herein do not necessarily state or reflect those of the United States Government or any agency thereof.

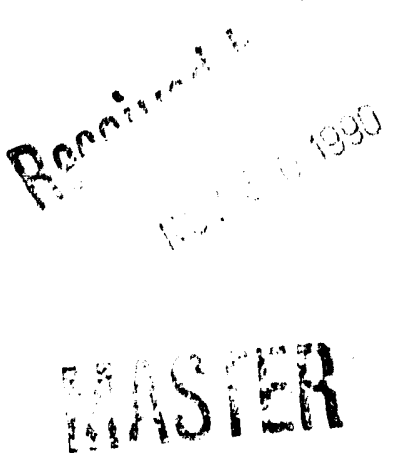




\section{TABLE OF CONTENTS}

I. Overview. ............................

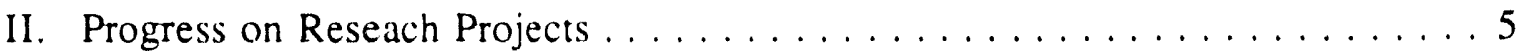

A. LAMPF E 1072, pp Elastic Absolute Cross Section Measurement . . . . . . . . . 5

B. LAMPF E876, Spin Transfer Measurements in np Elastic Scattering . . . . . . . 9

C. TRIUMF E372, Single Pion Production in $n$ p Scattering . . . . . . . . . 13

D. LAMPF E1097, Single Pion Production in $n$ p scattering . . . . . . . . 17

E. Fermilab E683, Photoproduction of Hign Pt Jets in the Wide Band Beam of the Tevatron . . . . . . . . . . . . . 23

F. BNL E791, Search for $\mathrm{K}_{\mathrm{L}}{ }^{\mathrm{o}} \rightarrow \mu \mathrm{e}, \mathrm{K}_{\mathrm{L}}{ }^{\mathrm{o}} \rightarrow$ ee $\ldots \ldots \ldots \ldots \ldots \ldots$

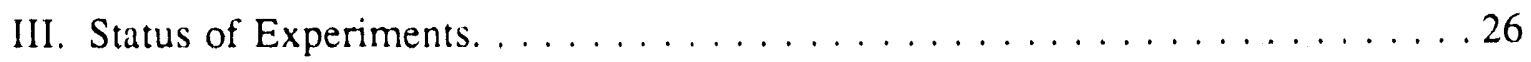

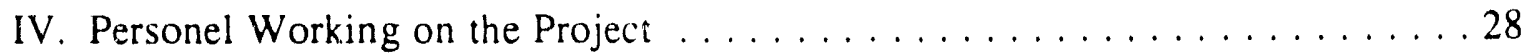

V. Publications and Presentations . . . . . . . . . . . . . . . . . . . . . 29

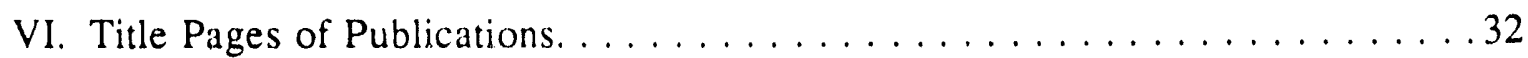


Riley - DOE 1990: Page 1

\section{MEDIUM ENERGY MEASUREMENTS OF N-N PARAMETERS}

\section{Overview}

We report here progress made for the three year period January 1,1988 , to December 31, 1990, for the Department Of Energy Three-Year Grant No. DE-FG0588ER 40446, third year. A major part of the work has been and will continue to be associated with research done at the Nucleon Physics Laboratory (NPL) at the Los Alamos Meson Physics Facility (LAMPF). The aim of the experimental program is the determination of the nucleon-nucleon amplitudes at medium energies. The required data include both elastic and inelastic experiments, and in addition the measurement of polarization and polarization transfer parameters. The measurements can be broadly categorized into those of (a) proton-proton elastic scattering, which probe the isospin-1 elastic channel, (b) neutron-proton elastic scattering, which allow measurements of isospin-0 amplitudes, (c) proton-proton inelastic scattering, and (d) neutron-proton inelastic scattering.

The LAMPF nucleon-nucleon ( $N-\mathrm{N}$ ) program was reviewed and a series of recommendations were made by Harvey Willard in the summer of 1988 . The review followed N-N working group meetings of LAMPF $\mathrm{N}-\mathrm{N}$ experimental teams (in which we played a role) held during the summer of 1987 at the request of Dr. G. Garvey, director of LAMPF, and C. Richardson, DOE Program Manager for Medium Energy Nuclear Physics. The Willard recommendations were in substantial agreement with the findings of the working group meetings, and are summarized below.

1. The LAMPF N-N program should be completed to the level of accuracy achievable with currently available techniques in a timely manner.

2. It is strongly recommended that cycle 52 be extended to September 30, 1988, to allow running at additional energies. This would allow completion of key $\mathrm{N}-\mathrm{N}$ experiments in an expeditious manner.

3. It is recommended that upon successful completion of PAC approved p-p elastic scattering experiment through 1072 (including analysis and publication) the proton-proton elastic scattering program at LAMPF be declared finished.

4. Strong encouragement should be provided by LAMPF management to ensure that all existing n-p data be aralyzed and published in a more timely manner.

5. Highest priority should be given to early completion of the crucial n-p elastic scattering spin transter experiments at 650 and $800 \mathrm{MeV}$ (E876).

6. Very high priority should be given to carrying out LAMPF $\Delta \sigma_{\mathrm{T}}(\mathrm{np})$ measurements (E960U).

7. It is recommended upon successful completion of PAC approved n-p elastic scattering experiments through E961 the neutron-proton scattering program at LAMPF should be considered finished.

8. Increased involvement of theorists in the $\mathrm{N}-\mathrm{N}$ program should be strongly encouraged at the present stage of developnent. 
9. Measurement of asymmetry and spin correlation parameters for $n p \rightarrow p p \pi$ (P1097) should be given serious consideration after the issues raised by the LAMPF PAC $(1 / 88)$ have been addressed.

10. Strong efforts should be made to ensure that the ANL group continue to play their catalytic role in the $N-N$ program at LAMPF.

\section{The LAMPF in-house effort in the $N-N$ program should be strengthened.}

12. An in-depth re-appraisal should be carried out in 1991 to assess the state of knowledge of the N-N interaction at LAMPF energies.

In making the above recommendations, Harvey Willard advocated that a schedule for experimenters developed by the experimental teams be followed. Two schedules had been proposed: an "Optimized Plan," and a "Constant Effort Plan," and are given below. (Willard advocated that the "Optimal Plan," estimated to require an additional 2 to 3 manyears of effort per year over the "Constant Effort Plan," be followed. )

1988

$$
\begin{aligned}
& \text { Optimized Plan } \\
& \text { E960 } \Delta \sigma_{L}(n p) \\
& \text { E1072 d } / \mathrm{d} \Omega(p p)
\end{aligned}
$$

1989

1990

1991

1992

1993

$$
\text { E960U } \Delta \sigma_{T}(n p)
$$

$$
\begin{aligned}
& \text { E961U } C_{N N}(n p) \\
& \text { or } n p \rightarrow d \gamma
\end{aligned}
$$

E876 Phase I $K_{i j}(n p)$

E1097 Phase I, np $\rightarrow$ pp $\pi^{-}$( $\mathrm{LH}_{2}$ Target)

E1078 $\mathrm{C}_{\mathrm{jj}}(\mathrm{pp})$ small angles: HRS/MRS

E876 Phase II K $\mathrm{K}_{\mathrm{ij}}(\mathrm{np})$

E1097 Phase II $n p \rightarrow p p \pi^{-}$

$$
\begin{aligned}
& \mathrm{np} \rightarrow \mathrm{d} \gamma(\mathrm{MRS}) \\
& \text { or } \mathrm{pp} \rightarrow \mathrm{np} \pi^{+} \text {(MRS) } \\
& \text { or other inelastic }
\end{aligned}
$$

Constant Effort Plan
Same
Same

(Analysis Only)

Same
Same

Same

$\mathrm{E} 1097 \mathrm{np} \rightarrow \mathrm{pp} \pi^{-}$ -..-.

E961U C $\mathrm{CNN}_{\mathrm{N}}(\mathrm{np})$

or $n p \rightarrow d \gamma$ (MRS)

or other inelastic

As one of the LAMPF experimental N-N teams, much of our efforts during the past 3 years, 1988-90, have been direced towards fulfilling the goals set out in the above schedules, at least to the extent of thuse experiments with which we have been involved. A major part of this progress report, therefore, reflects progress of the LAMPF $\mathrm{N}-\mathrm{N}$ experimental teams in carrying out the experiments discussed in the Willard report.

At this time, near the end of 1990 , the tentative schedule set out in the "Constant Effort" Plan has been followed rather well. E1072 measurements were carried oui during the summer of 1988. Data analysis is not yet complete, but it appears that the goal of $p-p$ differential cross section measurements at the $1 \%$ level at LAMPF energies has been achieved. The measurements of $E 876$ (polarization transfer, $\mathrm{K}_{\mathrm{ij}}(\mathrm{np})$ ), have required a much more intense polarized neutron beam than has been available in the past, and therefore have needed the high-intensity optically pumped polarized ion source, OPPIS, which has just become fully operational in 1990 at LAMPF. By the end of the summer, LAMPF was routinely delivering $800 \mathrm{MeV}, 400 \mathrm{nA}$ of polarized proton beam to 
our $\mathrm{LD}_{2}$ neutron production target, with polarization $>60 \%$, in comparison with polarized beam intensities of $10 \mathrm{nA}$ a decade ago. In spite of an accident on July 4,1990 , in which our 15 liter $\mathrm{LH}_{2}$ target was destroyed (and three students were injured), by the end of the run cycle on October 17, 1990 we were able to complete Phase l of E876, the measurement of the four spin-transfer parameters at $800 \mathrm{MeV}$. We plan to complete Phase 2 of the experiment in 1991. Phase I of E1097 has not yet been started, and although we plan to carry out detector tests during 1991, we do not expect to carry out Pha'e I until 1992, and Phase II in 1993, one year behind the recommendations of the Willard report. E960U has not been carried out; the requisite manpower has just not been available (The University of Texas was not a participant in E960). The LAMPF in-house effort in the N-N program has been somewhat strengthened by the addition of a research scientist, Dr. Ivan Supek. Further, the entire LAMPF staff should be commended for the achievements of OPPIS, which now makes possible a whole program of $n-p$ experiments. How'ever, it is clear that in the past three years the strengths of the experimental groups involved in $\mathrm{N}-\mathrm{N}$ experiments at LAMPF have, on the average, decreased in the amount of manpower available.

Our LAMPF work, and in fact our major commitment during the next few years will emphasize n-p ine'astic polarization measurements, LAMPF 1097, "Single Pion Production in np Scattering." Crucial to this experiment is the design and construction of a cylindrical drift chamber which will surround the target, and much of our present efforts are involved with the construction of this chamber. The schedule for this experiment calls for testing of our chamber at LAMPF during the summer of 1991. Phase I running, which will employ an unpolarized liquid hydrogen target, will be carried out during the summer of 1992, and phase II running, using a polarized (frozen spin) target, in 1993.

During the past three years we have been deeply involved in a neutron-proton inelastic scattering experiment at TRIUMF, (E372) "Single Pion Production in np Scattering," designed to measure the cross section and analyzing powers for the reaction $\mathrm{np} \rightarrow \mathrm{pp} \pi^{-}$. The E372 measurement is the same as will be carried out in LAMPF 1097, phase I, but at lower energies. E372 was much delayed by unexpected difficulties with the TRIUMF optically pumped ion source, which only three years ago appeared to be about one year ahead of the LAMPF ion source OPPIS, but which now lags behind OPPIS. However, we have succeeded in obtaining a large amount of data during a run in September 1990, and believe that we can complete the measurements during our next run, in November 1990. The Ph. D. dissertation of Mark Bachman will be based on E 372 data.

During the past year we also took part in data runs at Brookhaven National Laboratory, "Study of Very Rare $K_{L}{ }^{\circ}$ Decays," (BNL 791). Data taking for this experiment is now completed, but a proposal for a new generation rare-decay measurement has been written (BNL 871), and we expect to participate in this new experiment during the next few years. We are also participating in a Fermilab experiment, Fermilab E683, "Photoproduction of High $\mathrm{p}_{\mathrm{t}}$ Jets in the Wide Band Beam of the Tevatron" (M. Corcoran, of Rice University, Spokesperson).

During the past three years we have greatly increased the fraction of our research being carried out at the University of Texas, especially research related to software development and data analysis. We acquired two additional graphics terminals in 1989, and were able to obtain full-time usage of a VAX 3100 work: n. We purchased a larger (300 Mbyte) disk for the workstation to allow us to use it with use LAMPF data acquisition program $Q$, and with our TRIUMF data acquisition and analyses programs. We are presently starting to use an IBM RISC system/6000 which has become available in the department of Physics, and would like to purchase one for full-time usage. We have also 
made extensive use of the Physics Department machine shop, having fabricated here the large scintillator detector stand ("Texas Snowplow") for TRIUMF E372, and more recently, the test chamber for E1097, the detector end-plates for E1097, and the mandrils used for fabricating the carbon cylindrical chamber walls.

In the following section we describe recent progress on three LAMPF experiments, E1072, E876, and E1097, one TRIUMF experiment, E372, one BNL measurement, E791, and one FNL experiment, E683. We are deeply involved in all phases of the three LAMPF and the single TRIUMF experiments. Our degree of involvement with both the BNL and with the FNL experiments has been much less significant. 


\section{PROGRESS ON RESEARCH PROJECTS}

\section{A. LAMPF E1072, pp Elastic Absolute Cross Section Measurements}

The aim of Experiment 1072, which was carried out during the summer of 1988, is to measure the absolute $p$-p differential cross section at LAMPF energies to an absolute accuracy of $1 \%$. Measurements were made over an angular range of about $13^{\circ}$ to $41^{\circ}$ in the laboratory frame, at energies of $500,575,642,733$, and $800 \mathrm{MeV}$. The data are being analyzed by a joint effort from LAMPF, the University of Texas (Kok Heong McNaughton), and Texas A\&M Universities. The following is a short description of the current state of this analysis.

Three different targets were used in this experiment. A liquid hydrogen target $\left(\mathrm{LH}_{2}\right)$ was used for most of the data; polyethylene $\left(\mathrm{CH}_{2}\right)$ targets and water targets $\left(\mathrm{H}_{2} \mathrm{O}\right)$ were used to search for systematic errors. All of the $\mathrm{LH}_{2}$ data have been replayed at all 5 energies. None of the $\mathrm{H}_{2} \mathrm{O}$ data has been replayed and only one energy of the $\mathrm{CH}_{2}$ data has been replayed and still needs to be compared with the liquid target data.

There were basically two detector setups for this experiment: one (a combination of wire-chambers and scintillators) to detect scattered particles, and the other (Hamamatsu beam counters) to determine the absolute beam normalization (see fig. 1). Much of the work done on the beam counter system analysis can be found in two papers by E. Gulmez et al. 1 The "scattered particle analysis" involves many factors, all of which need to be looked at separately. Two Multi-Wire Proportional Chambers (MWPCs) and three drift chambers (MWDCs) were used on each arm of this two arm experiment to detect the scattered particle and the recoil particle. All of the detectors must be well understood to reduce systematic errors. For instance, the efficiencies of the MWPCs have been measured in two different ways; the two measurements have been found to be in good agreement with one another. One method makes use of the drift chambers to determine the track of a good p-p scattered event. This event can be used to determine if the MWPCs give the same result. Alternately, three of the four MWPCs can be used to determine a kinematically good event. From the three, the expected hit position of the particle in the fourth chamber can be determined and compared to the actual hit position. From these methods, we have found that the overall MWPC efficiency is generally 98 to $99 \%$. Most of the inefficiency of the MWPC system is caused by two bad wires in one plane of one chamber. (The efficiency of the chambers will also decrease as the event rate increases, but this experiment did not run at a high enough rate to see this effect. ) A plot of the overall MWPC efficiency vs. beam rate indicates that the chamber efficiency is fairly constant over the range of our experiment. 


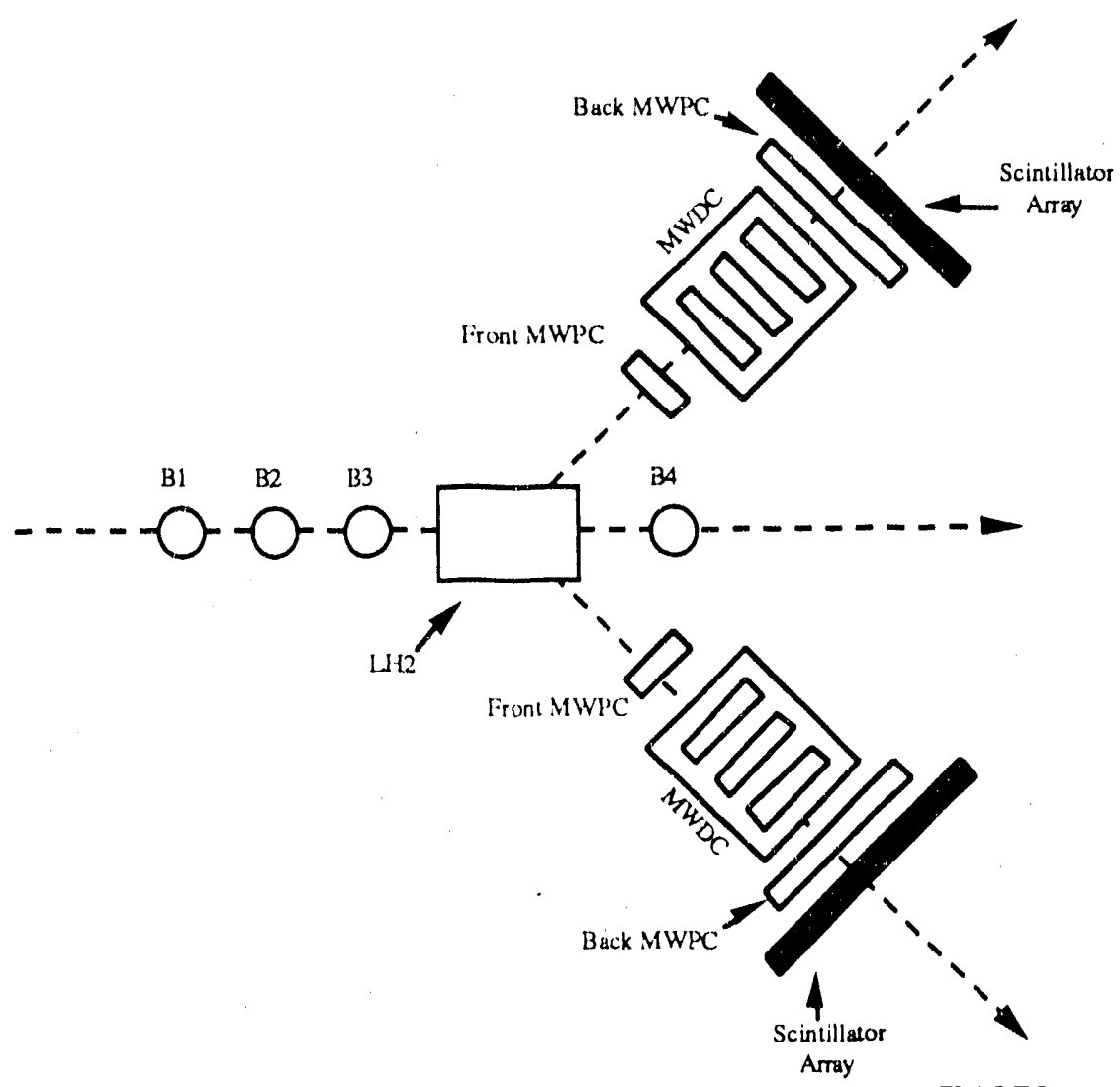

Figure 1: Experimental layout for LAMPF E1072

Part of the efficiency determination as well as the cross section calculations depend on how a good event is defined. An initia! criterion for a good event is that it is kinematically well defined in the chambers. This includes a $\phi$-coplanarity cut and a $\Delta \theta$ cut. $\phi$-coplanarity is a requirement that the two particles are in the same scattering plane as defined by conservation of momentum. The $\Delta \theta$ cut is a restriction defined by conservation of momentum as well as energy. Furthermore, the $\phi$-coplanarity values are normalized by $\sin (\theta)$ to give $\Delta \phi$. This gives the shape of the $\Delta \phi$ spectra to be about the same as the $\Delta \theta$ spectra, with the same uncertainty, $\sigma$. The sum of the square of $\Delta \theta$ and the square of $\Delta \phi$ is defined as RSQ. Cuts are then placed on RSQ to define a good event. The RSQ cut gives a circular cut rather than a square cut in the binning of the events. These cuts are determined by a Monte Carlo program to be about 5 standard deviations. Anything outside of the cuts are considered to be background and corrections for the background as well as for multiple scattering are made based on the Monte Carlo simulations.

Much work has gone into the determination of the solid angle, which is essentially defined by the chamber geometry. However, the chambers aren't all exactly parallel to one another and this can cause an error both in the angle calculations and the solid angle. In addition, the chambers aren't actually planes, but are slightly distorted. Most of the distortion effects in our experiment can be traced to one chamber and fortunately only affect measurements close to the edge of the chamber. In fact, the area of concern is partly outside the acceptance area of the chamber system and does not affect the solid angle.

We have also been concerned with the determination of the actual target size, which changes with temperature and pressure. From this and the density of the liquid hydrogen used in the target, corrected for temperature and pressure effects, we can calculate the target 
thickness in number of particles per millibarn, which goes into the tetermination of the final cross-section values.

Data acquisition live-time was monitored throughout the experiment in several different ways using various combinations of the coincidence signals of the beam counters and the scintillator planes. These are all in good agreement. Currently, work continues in analysis of the background and study of beam attenuation in the target and surrounding materials. Preliminary results of the cross section vs. angle at 575 and $642 \mathrm{MeV}$ are plotted here with Arndt's values from his phase shift analysis program (SM89). These numbers are in excellent agreement with Arndt results.

1 E. Gulmez, A. G. Ling, C. A. Whitten, Jr., J. F. Amann, M. W. McNaughton, T. Noro, D. L. Adams, V. R. Cupps, R. D. Ransome, G. Glass, A. J. Simon, K. H. McNaughton, and P. J. Riley, "Accurate Intensity Measurements for proton Beams with a $201 \mathrm{MHz}$ Structure," Nuclear Instruments and Methods A, (to be published). 

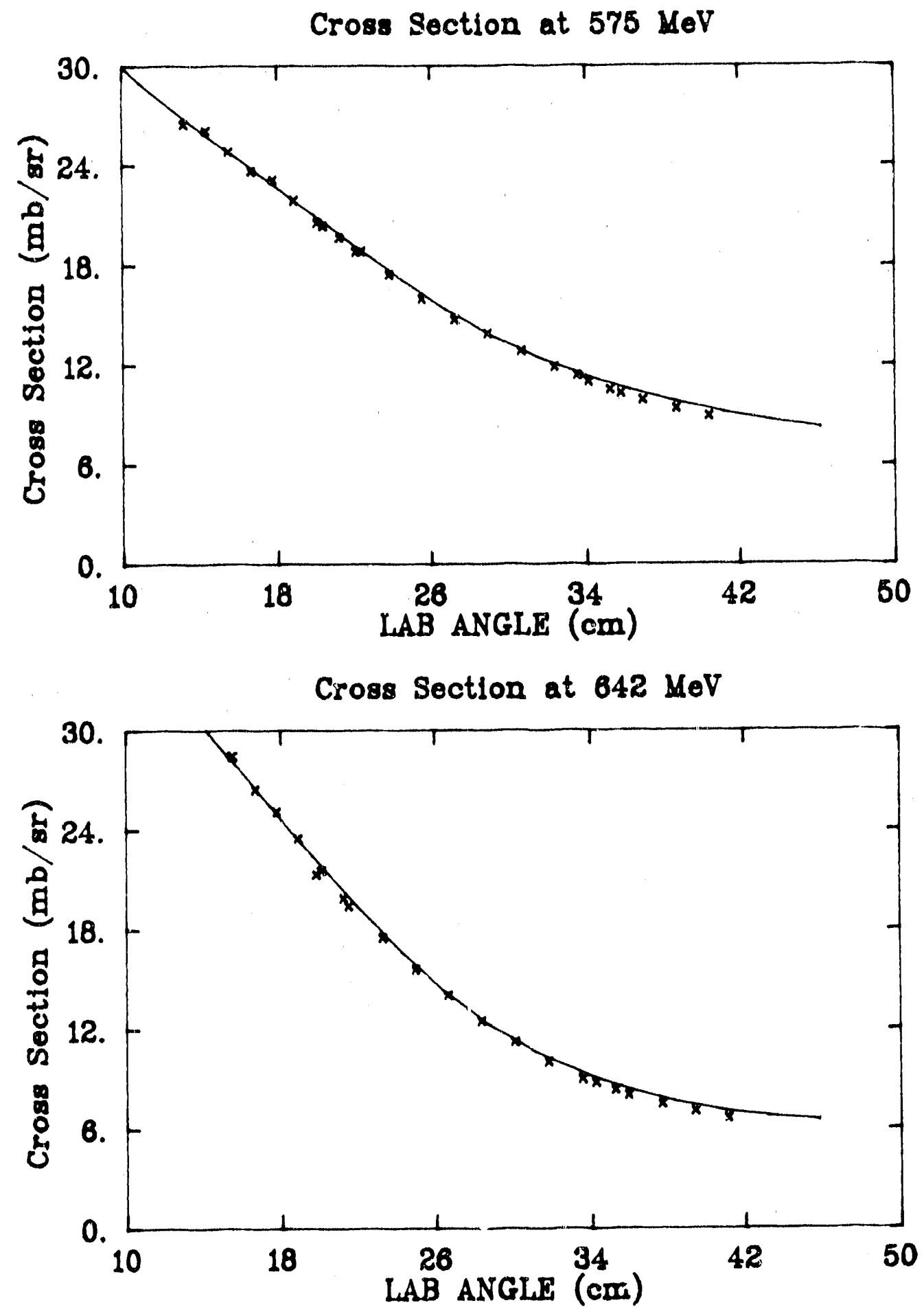

Figure 2: Experimental and predicted cross sections for E1072 


\section{B. LAMPF E876, Spin Transfer Measurements in np Elastic Scattering}

For some time now it has been suspected that the nucleon-nucleon isospin-zero $(\mathrm{NN} I=0)$ phase shifts are seriously in error. Our preliminary results from experiment E876, a measurement of the angular distribution of the spin transfer coefficients for np elastic scattering at 650,733 , and $800 \mathrm{MeV}$, has confirmed these suspicions. NN phase shifts to the global data base are maintained by Arndt ${ }^{1}, \mathrm{Bugg}^{2}$, and Bystricky ${ }^{3}$, all of whom agree that the NN I=0 phase-shifts are inadequate, especially above $500 \mathrm{MeV}$. Arndt ${ }^{4}$ writes that above $500 \mathrm{MeV}$ the NN $\mathrm{I}=0$ phase shifts are "essentially undetermined". Experience from the NN I=1 measurements demonstrated that the phase shift analysis remained unstable until sufficient data existed for a complete determination of the 5 complex NN amplitudes, and until then the statistical uncertainty (corresponding to an increase of one in the $\chi^{2}$ ) was far too optimistic. Changes of five standard deviations were found ion the early $I=1$ predictions. Even larger changes are now being observed in the $\mathrm{I}=0$ predictions.

Bugg specifically identified the spin-transfer coefficients as being necessary to fix. the phase-shifts, and in response to this, LAMPF experiment 876 was proposed, and was the major incentive for the high intensity polarized source (OPPIS) at LAMPF. 1990 was the first year of full OPPIS production and the first year of running for E876.

The apparatus, shown in Fig. 3, is essentially the same as had been used in previous LAMPF experiments $5,6,7$. Briefly, polarized protons are focussed onto a liquid deuterium target and neutrons, polarized by spin transfer, are collimated at zero degrees and directed onto a 15 liter liquid hydrogen target (the largest ever used at LAMPF). Scattered neutrons are detected in a large high-efficiency neutron detector. Recoil protons are detected in the Scylla spectrometer, which serves a dual purpose: 1) to measure the momentum and 2) to precess the recoil protons from L-spin to $\mathrm{N}$-spin. Finally, the proton spin is analyzed in the Janus polarimeter.

The experiment uses a new (for us) Camac readout system called FERA. Extensive work went into readying the detectors in the spring of 1990, even before the beam went on. Detectors were tested with sources and calibrated as well as possible without beam. The software relating to the new FERA readout were written and tested extensively. Simulations were done to understand as much of the system as we could before we took data.

The first data were taken in the middle of May with a $\mathrm{CH}_{2}$ target to study detector alignment, rates and background. At the end of May, the $\mathrm{LH}_{2}$ target was ready and the experiment ran smoothly with few interruptions through all of June. We were able to take data with the neutron beam at $487 \mathrm{MeV}$ for $6 \mathrm{lab}$ angle settings in ooth the $\mathrm{L}$ and $\mathrm{S}$ configurations, measuring all 5 observables $K_{L L}, K_{L S}, K_{S L}, K_{S S}$ and P. While datataking was in process in the BR counting house using the MicroVax II, we had sufficient manpower to allow us to do some replay of the freshly taken data in the adjacent MRS counting house (using a MicroVax III) as well as through batrh jobs in the DAC so that we were able to obtain some preliminary replayed data of these observables. 


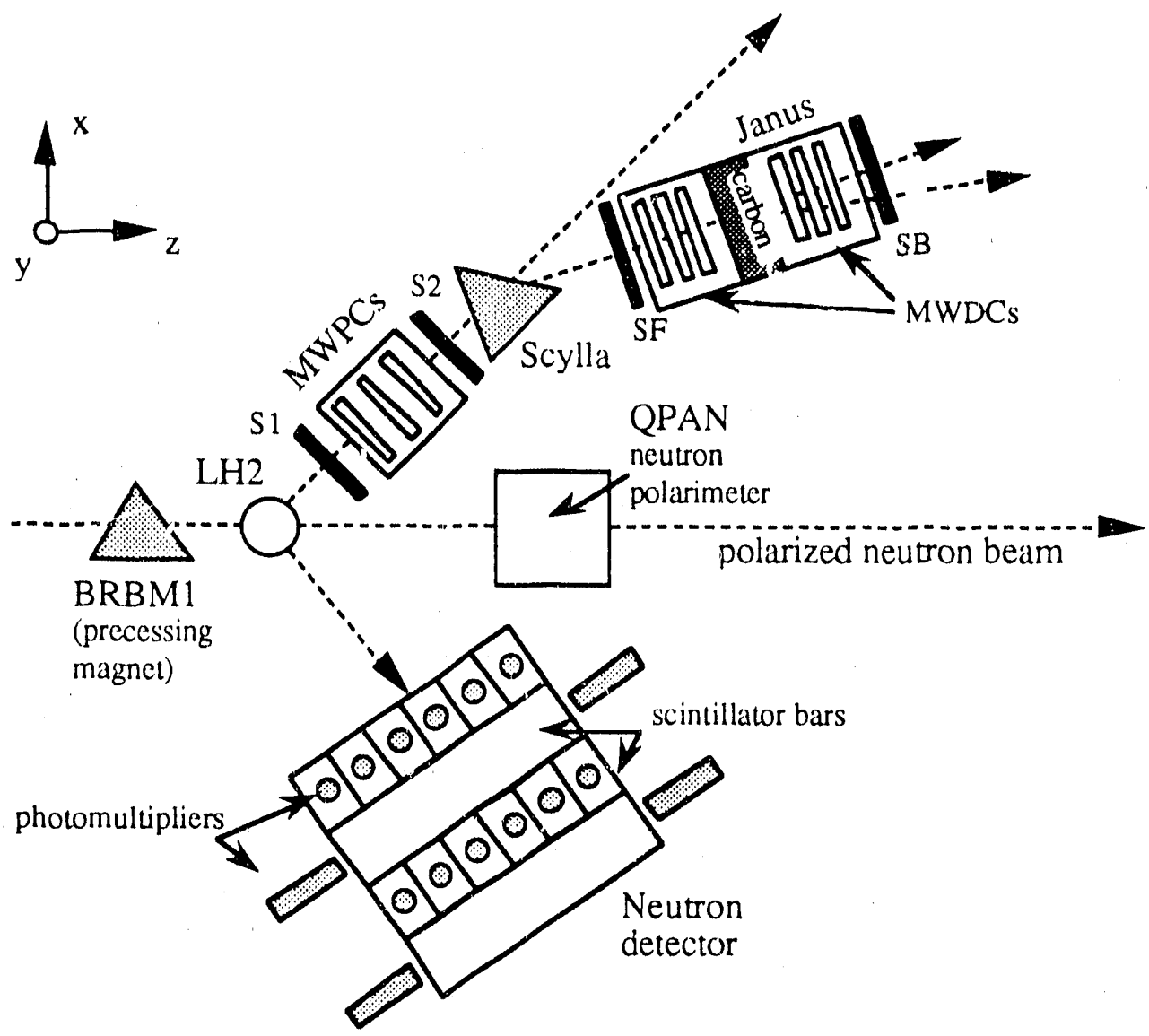

Figure 3: Experimental setup for LAMPF E876

We changed to $8 \mathrm{CJ} \mathrm{MeV}$ neutron beam energy on June 30 and took data at $60-, 50$ and $30^{\circ} \mathrm{lab}$ (proton) angle in both the $\mathrm{S}$ and $\mathrm{L}$ configuration. When we were changing the angle from $30^{\circ}$ to $10^{\circ}$ on July 4 an accident occured which destroyed the $\mathrm{LH}_{2}$ target. (See LAMPF's Investigation Report: August 1990 HSE 90-4). Because of the accident, datataking was interrupted by more than 2 months while an investigation was conducted, damage accessed, and detectors that were destroyed or damaged were rebuilt. Some efforts were also diverted to studying the STARBURST system, which we hope to use in the 1991 phase of the experiment. Immediately after the accident it was estimated that the rebuilding of the $\mathrm{LH}_{2}$ target alone would require several months.

During August, we took some data with beam using carbon and $\mathrm{CH}_{2}$ targets to study background and alignment. We are satisfied that the alignment of the new setup is as good as the previous one. In September, we were able to use a 3-liter target filled with $\mathrm{LD}_{2}$ to study the neutron beam polarization and to take E876 data at forward scattering angles. The use of an $\mathrm{LD}_{2}$ neutron scattering target, as well as an $\mathrm{LD}_{2}$ neutron production target allowed us, by measuring the polarization of the $0^{\circ}$ quasi-elastic $(n, d)$ protons, to effectively measure $\left[K_{L L}\right]^{2}$ at $800 \mathrm{MeV}$. We could therefore deduce $\left[K_{L L}\right]_{p, d}$ (the mechanism through which a polarized neutron beam is obtained at LAMPF) from the polarization of the incident proton beam without needing to know the $(n, p)$ analyzing power. The preliminary value for $K_{L L}=0.68 \pm 0.03$, which compares with Chalmers ${ }^{8}$ value of $0.60 \pm 0.05$ and with Riley's 9 value of $0.64 \pm 0.05$. We have used the value of $K_{L L}=0.68 \pm 0.03$ for our on-line calculations of polarization transfer values. 
LAMPF staff completed the rebuilding of the 15 liter target by the end of September, well ahead of the time earlier estimated, and installed it successfully at the beginning of October. The refurbished 15 iter $\mathrm{LH}_{2}$ target was used successfully until the end of the experimental run, on October 17, 1990. Preliminary $800 \mathrm{MeV}$ on-line polarization transfer data are shown in the figures. The solid lines are the Arndt phase shift predictions $(\mathrm{C} 800)$. The dotted lines indicate the uncertainty corresponding to an increase of one in the $\chi^{2}$. Several conclusions can be drawn:

1. These new data are internally consistent. For example, the center of mass relation 7 $\mathrm{K}_{\mathrm{sl}}=-\mathrm{K}_{\mathrm{ls}}$, where the spins have been transformed to the center of mass, checks out well.

2. There are serious systematic discrepancies with the existing phase-shift predictions. For example, near $122^{\circ} \mathrm{cm}$ Arndt predicts $\mathrm{K}_{\mathrm{LL}}=-0.742 \pm 0.011$, while we measure $\mathrm{K}_{\mathrm{LL}}=-0.96$, with a statistical uncertainty of 0.03 and a preliminary systematic uncertainty ii 0.04 .

3. The relationship $K_{L L}{ }^{*} K_{L L}+K_{L S} * K_{L S}<1.0$ plares an upper linit on the polarization of the neutron beam. This same neutron beam has been used in all previous np measurements at LAMPF. If we use the Chamers calibration of the neutson polarization, then our me isurements would imply that $K_{L L} K_{L L}+K_{L S} * K_{L . S}=1.15 \pm 0.03$. The implication is that the previous data w:.l need to be normalized by $15 \%$. Note that this renomalization is consistent with the $13 \%$ normalization predicted by $\mathrm{Bugg}^{2}$.

4. Careful off-line replay of pur data is proceeding, and should result in improved uncertainties.

In summary, Phase 1, the measurement of the four spin-transfer observables $K_{L L}$, $\mathrm{K}_{S S}, \mathrm{~K}_{\mathrm{SL}}$, and $\mathrm{K}_{\mathrm{LS}}$ at $800 \mathrm{MeV}$ have been completed successfully. In 1991 we plan to complete Eó76, measuring these four observables at 640 and $725 \mathrm{MeV}$ and measuring the observables $\mathrm{K}_{\mathrm{NN}}$ and $\mathrm{P}$ at 640,725 , and $800 \mathrm{MeV}$.

1 R. A. Arndt, et al., Phys. Rev. D35, 128, (1987).

D. V. Bugg, Phys. Rev. C $\$ 1,2708,(1990)$.

3 J. Bystricky, C. Leluc, and F. Lehar, J. Phys. 48, 199, (1987).

4 R. A. Arndt, Phys. Rev. D37, 2665. (1988).

${ }^{5}$ R. Ransome et al., NIM 201, 309, (1982).

6 M. W. McNaughton et al., Phys. Rev. C\$1, 2809, (1990).

7 C. L. Hollas et al., Phys. Rev. C30, 1251, (1984).

8 8. J. S. Chalmers et al., Fiys. Letters 153B, 235, (1985).

${ }^{9}$ P. J. Riley, et al., Physics Letters 103B, 313. (1981). 

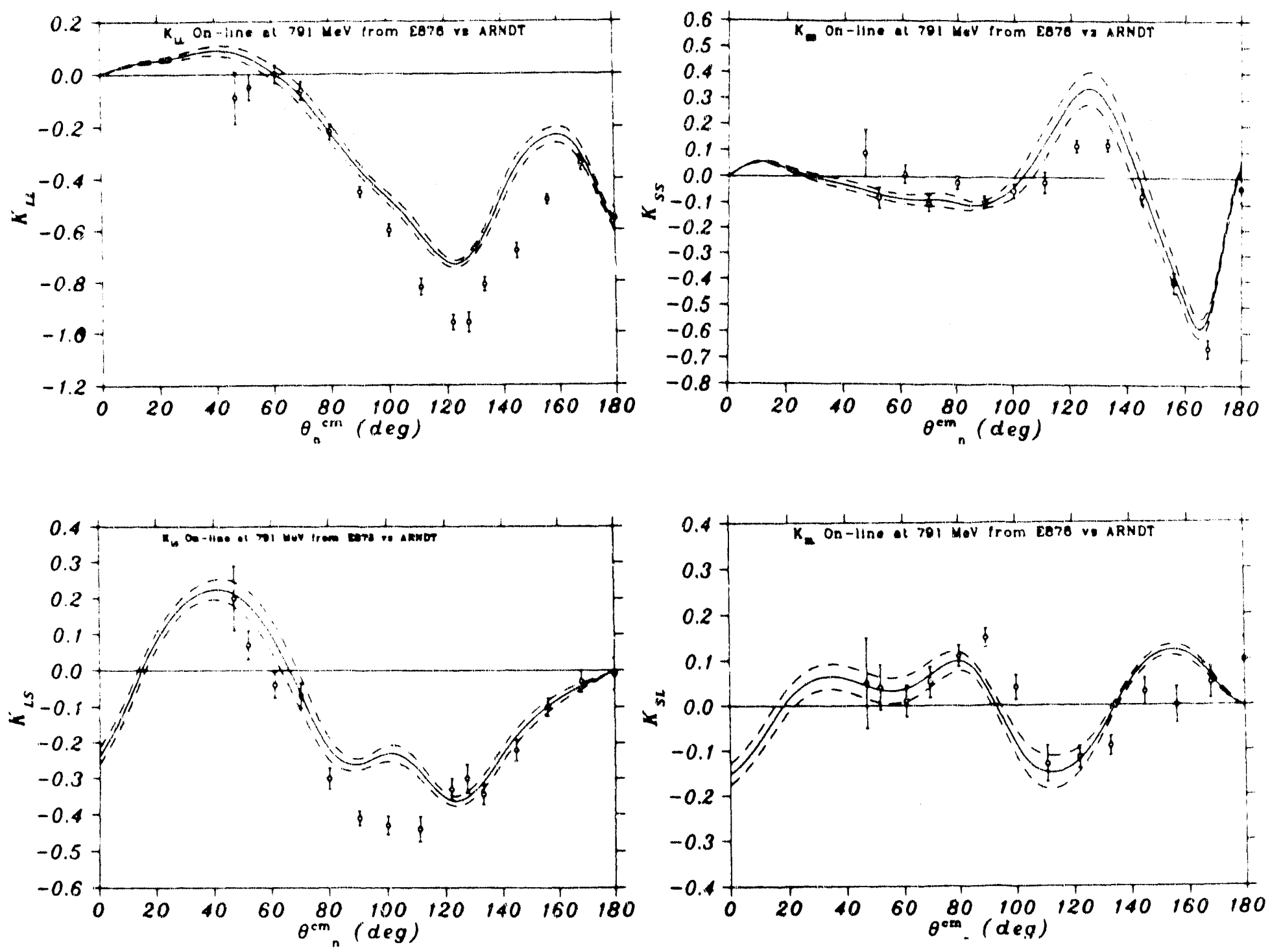

Figure 4: Spin transfer measurements at $791 \mathrm{MeV}$ 


\section{TRIUMF Experiment 372, Single Pion Production in nẹ Scattering}

The purpose of TRIUMF experiment E372 is to measure the differential cross section and analyzing powers $A_{x}, A_{y}$, and $A_{z}$ for the reaction $n p \rightarrow p p \pi^{-}$at $470 \mathrm{MeV}$. Knowledge of these observables will help us to understand the role of nonresonant amplitudes in the fundamental strong process of pion production. TRIUMF E372 uses an intense polarized neutron beam which is directed by means of a collimator on to a liquid hydrogen target. The trajectories of the two protons are measured in a detector directly downstream of the hydrogen target. From the proton trajectories and knowing the energy of the neutron beam, we can reconstruct the kinematics for each pp $\pi^{-}$event. From this information, we bin our data to extract the observables and compare to theoretical models.

The TRIUMF cyclotron is capable of providing polarized protons of up to 500 $\mathrm{MeV}$. These protons are directed on to a liquid deuterium target where they undergo a charge exchange reaction $2 \mathrm{H}(\mathrm{p}, \mathrm{n}) 2 \mathrm{p}$ with the deuterium. The polarization transfer for this reaction is maximum at $9^{\circ}\left(\mathrm{R}_{\mathrm{t}}=.71\right)$, and this is the angle at which the neutron collimator sits. For our experiment, we used a new ion source "I4" which is capable of producing over $10 \mu \mathrm{A}$ of polarized protons. I4 was crucial to E372 because the usual Lamb shift ion source at TRIUMF ("I2") produces protons with a maximum current of $1 \mu \mathrm{A}$. The usual beam tune at TRIUMF produces beam pulses $\sim 2.5$ nanoseconds wide, every 43 nanoseconds. We calculate the energy of the neutron beam by timing our events with respect to the RF of the proton beam. A $2.5 \mathrm{nsec}$ uncertainty corresponds to an uncertainty in our beam energy of $\sim 90 \mathrm{MeV}$ which is not good enough for us to reconstruct the kinematics of our events. I4, which uses an optically pumped sodium cell to create the intense beam of polarized protons, produces enough beam so that a special beam tune ("MERO") can be done. The MERO tune restricts the phase space of the beam so that over $90 \%$ of the beam is lost, but results in pulses of $0.5 \mathrm{nsec}$ duration. This time tune corresponds to an uncertainty of less than $15 \mathrm{MeV}$ in our beam energy, adequate for event reconstruction.

The diagram below illustrates the main components of the E372 detector. The basic triggering elements are the start scintillator S1 and the stop scintillator bars S2. The kinematics of the pp $\pi^{-}$reaction are such that no proton may leave the $\mathrm{LH}_{2}$ target at an angle of greater than $45^{\circ}$. The scintillator array (S2) contains 18 scintillator bars, each $200 \times 3 \times$ $10.2 \mathrm{~cm}^{3}$, with the axis of the cylinder horizontal and passing through the $\mathrm{LH}_{2}$ target. These bars are mounted $120 \mathrm{~cm}$ from the $\mathrm{LH}_{2}$ target and have almost full acceptance for all $\mathrm{pp} \pi^{-}$protons at an incident neutron energy of $438 \mathrm{MeV}$. Since at least twc viarged particles are detected in the pp $\pi^{-}$events, and since no other neutron-proton reaction can produce two charged particles, we use the basic criterion that two S2 bars fire for our trigger. The S2 array also acts as a spectrometer since we calcuate the momenta of the protons from their times of flight between the start scintillator and the bars. Between the scintillators and target are two large multiwire drift chambers, each containing six coordinate planes $(x, y, u)$ to track the final state particles. We also use multiwire proportional wire chamber's on the left and right sides of the target to detect pions which happen to scatter at large angles. In addition to these major components, we include a veto scintillator to veto charged particles in the neutron beam, a neutron profile monitor behind the assembly to monitor the beam position, calibration scintillators along the beam axis to calibrate the true center of our coordinate system, "button" scintillators at precise positions along the backs of the scintillator bars for timing calibrations, and two neutron polarimeters before and after our precessing magnets. 


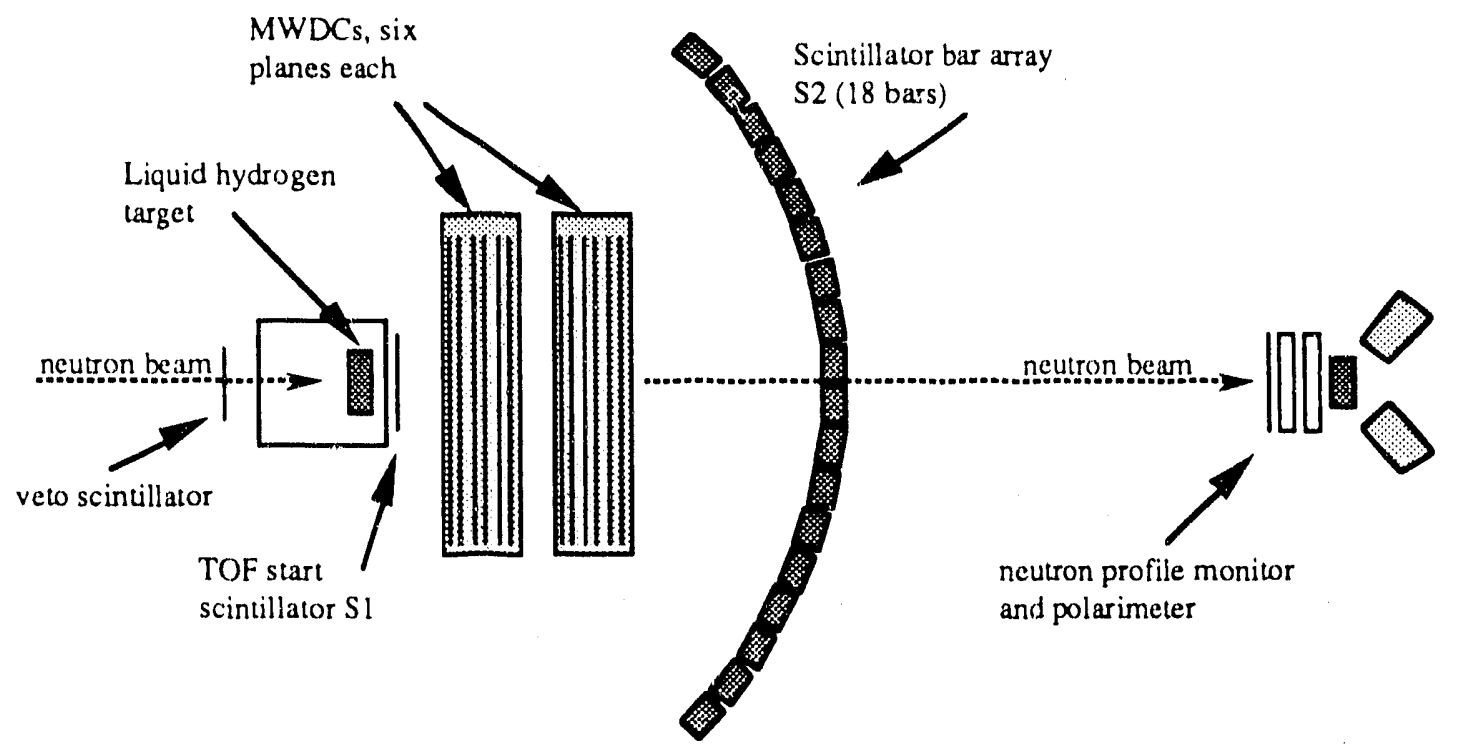

Figure 5: Experimental detector for E372

We spent a considerable amount of effort designing and constructing the detector for E372. We wrote and used a Monte Carlo simulation to help us optimize our experimental design and from initial studies it became clear that we would require at least a $250 \mathrm{psec}$ uncertainty $(\sigma)$ in bar timing to reconstruct the pp $\pi^{-}$events accurately. We found that the cylindrical design of the scintillator wall minimizes the occurrence of "punch through," where a single charged particle hits two scinillator bars simultaneously. With the design, we expect (from Monte Carlo) an acceptance of $-95 \%$ of phase space, the major losses coming from two protons hitting the same bar. We built the large; support structure for the S2 array here in Texas and shipped it up to TRIUMF where we ruassembled it and connected the scintillators and electronics.

We have also invested a great amount of time in software development and analysis for E372. Using Monte Carlo data initially, we began the development of algorithms for extracting the tracks from the MWDCs. It quickly became clear that "tracking" is a major problem. The analysis requires fast computers, since the pattern recognition needed for tracking takes a considerable amount of computer time. We also developed kinenatic event reconstruction codes for reconstructing the events by minimizing the $\chi^{2}$ in our measurements. After taking test runs, ive were able to analyze real data to determine the calibrations and limitations of our detector. We found that the timing resolution for the bars was on average $240 \mathrm{psec}(\sigma)$, better than the $250 \mathrm{psec}$ needed. The problem of "punch throughs" was reduced to a $4 \%$ probability per track. The main source of background seerns to come from gamma pair production in the walls of our target, resulting in an $\mathrm{e}^{+} \mathrm{e}^{-}$ pair hitting our scintillator bars. Most of this was eliminated in later runs by sharp cuts on timing spectra with respect to the proton beam bursts. In addition, we developed systematic and clean algorithms for calculating the calibration constants needed for all the detectors' TDCs and ADCs.

From the time when $E 372$ first began taking test data to the present, the acquisition computer for the experiment at TRIUMF has changer from an Eclipse computer to a VAX750 to a MicroVAX. Each change has brought about new challenges for the software. The Eclipse run an old acquisition software package called DACS which had very limited flexibility and almost no online diagnostic spectra. The switch to the new VAX. machines allowed us to use a newer (though not well tested) acquisition system called 
VDACS, coupled with an online histogramming package called LISA. We wrote the majority of online diagnostic software for LISA which we used while taking data on later runs at TRIUMF. This software was very useful as we were able to monitor most of the equipment used in our experiment and determine any problems quickly and without question. Furthermore, we used the RF timing spectra to make the cuts on the acquisition needed to remove the gamma induced events.

In January of 1990, E372 set up to run and take data. However, the I4 source failed to produce polarized beam. We took unpolarized data for calibration purposes and tested our equipment. There were some problems of "cross talk" associated with the large multiwire drift chambers. (We discovered we could correct for this in software after analyzing events offline. ) We also checked the efficiency of our trigger, electronics, and acquisition software. With the exception of the polarized source, everything about E372 worked reasonably well.

TRIUMF continued development of their I4 source through the summer of 1990. A 10 day E372 data run was made in September 1990. This time I4 worked much better, though not as quite well as we'd hoped. The beam tune (monitored by our fast timing scintillators) produced a nice $0.5 \mathrm{nsec}$ proton pulse, but the proton polarization fluctuated somewhat, being about $40 \%-50 \%$ on average. Furthermore, problems with the in-beam polarimeters lead to inconsistent readings of the true proton polarization at times. However, we were able to take 25 VCR tapes of data corresponding to 36 million events written to tape. Of these events, 12 million were with an empty target for background subtraction. We ran with the neutron beam in all three orthogonal projections: $N, S, L$ (normal, sideways, and longitudinal), $\sim 20 \%$ of events for $\mathrm{N}, \sim 30 \%$ of events for $\mathrm{S}$, and $\sim 50 \%$ events for $\mathrm{L}$. Figure 6 shows vertex reconstruction for some of our data. The target width is reproduced as is the upstream kevlar wall of the target flask. The Z-vertex reconstruction is poorer in resolution due to abundance of tracks with small slopes. The 3 -track events reproduce the vertex with greater resolution then the 2-track events, as one might expect. The success of our run in September allowed us to be approved for more beam time in November 1990 and February 1991. We will take more E372 data during the week of Thanksgiving, 1990, and in February 1991.

Our next step with E372 (in addition to the Thanksgiving and February runs) is to analyze the data. We have begun an initial look at the data to see if everything seems to work and to check calibrations. The University of Texas and the University of Manitoba both have copies of the data which we will analyze concurrently. The University of Manitoba is performing the tracking and making summary tapes; the University of Texas will reconstruct the kinematics of the events by $\chi^{2}$ minimization. Each institution will completely analyze a subset of the data independently to provide a cross check of the analysis. By breaking the analysis into two steps, we hope to increase the speed at which we can analyze the data.

From the beginning of this experiment, the University of Texas has been a major contributor of equipment, manpower and development. We have participated in every data run of importance and have actively assisted at all levels of development, from soldering electronic parts to building equipment to writing the analysis software to making decisions about the direction of the experiment. We intend to continue our commitment to making the measurements of pion production in the np irelastic reaction. 

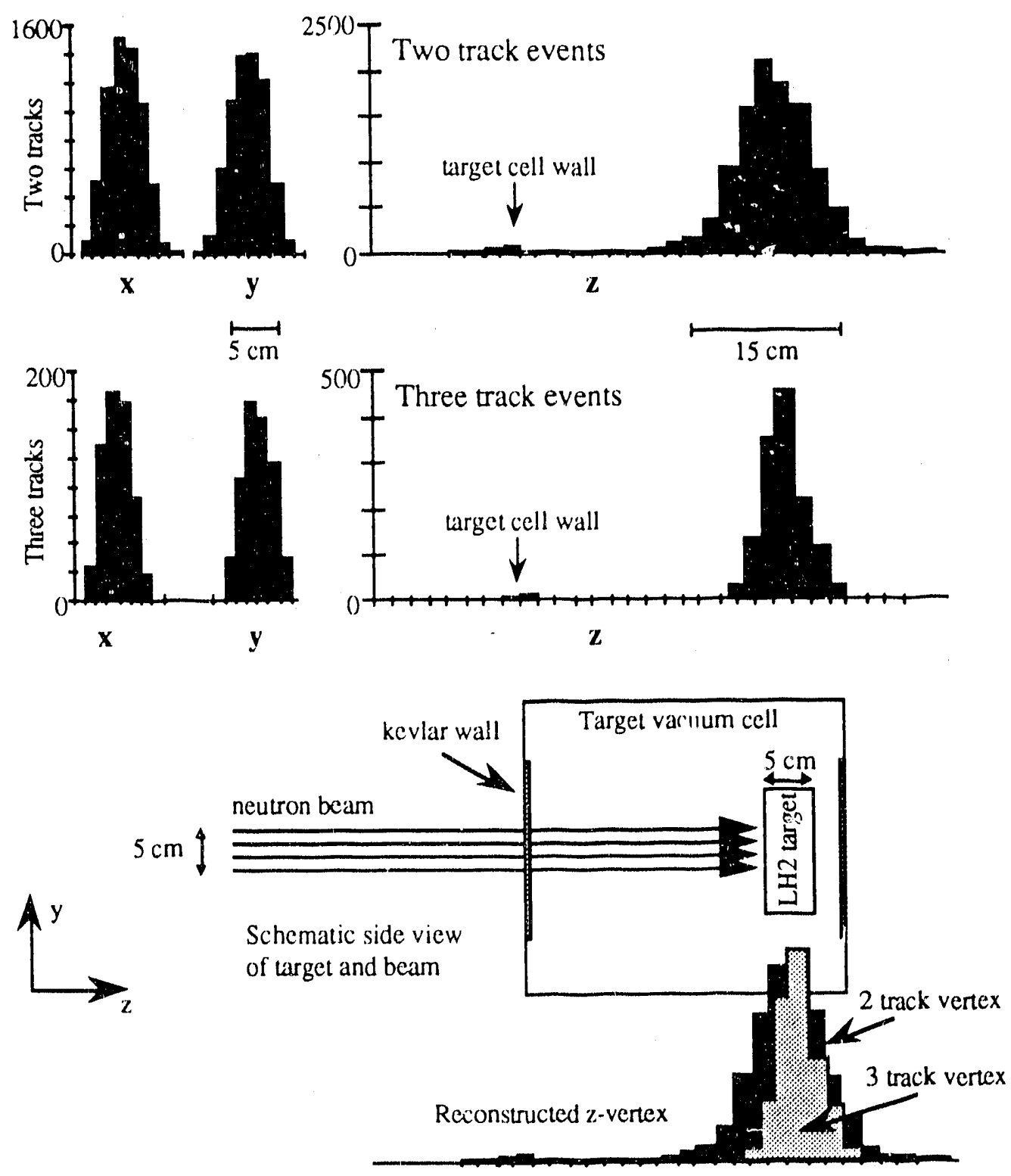

Figure 6: Vertex reconstruction from E372 tracking codes 
Riley - DOE 1990: Page 17

\section{LAMPF E1097, Single Pion Production in np Scattering}

\section{E1097: Overview}

In recent years, there has been a major effort in both the experimental and theoretical investigation of the coupled NN-NN $\pi$ problem. This set of reactions is one of the simplest inelastic processes available for study. It sheds light on the $N N$ and $N \pi$ interactions and is key to understanding pion production in more complex systems. There are four independent channels available in the $\mathrm{NN}-\mathrm{NN} \pi$ reaction. These channels can be expressed in terms of the total isospin of the initial (I) and final (I') state nucleons (I,I'): $(1,0),(1,1),(0,1),(0,0)$. The dominant channel for pion production is through the $(1,0)$ resonance, since the famous $\Delta^{++}(I=3 / 2, P=3 / 2)$ can proceed through this channel. The role of the non-resonant amplitudes, particularly the channel $(0,1)$ remains elusive. The reaction $\mathrm{np} \rightarrow \mathrm{pp} \pi$ may proceed through the $(1,1)$ and $(0,1)$ channels, which avoid the $\Delta^{++}$, making it an ideal reaction for studying the non-resonant contributions to pion production. The reaction is experimentally accessible, since all final state particles are charged, allowing efficient and precise determination of all outgoing trajectories.

LAMPF Experiment E1097 will measure the differential cross section, $\mathrm{d} \sigma / \mathrm{d} \Omega$, and the spin observables $A_{N}\left(, A_{L O}, A_{S 0}, A_{0 L}, A_{S L}, A_{N L}\right.$, and $A_{L L}$ for the single pion production reaction $n p \rightarrow p p \pi^{-}$at neutron beam energies in the range $500-800 \mathrm{MeV}$. These data should allow a first order partial wave analysis of the $I=0$ inelastic amplitudes. To accomplish this, E1097 will run in two separate phases. Phase I, planned for 1992, will use a polarized neutron beam directed on to a liquid hydrogen target; phase II, expected to run in 1993, will use a polarized hydrogen (frozen spin) target. During the summer of 1991 we plan to set up and test the major detector being built for the experiment, a large acceptance cylindrical drift detector. The E1097 Spokesmen are N. E. Davison (Manitoba), D. L. Adams, (Rice), G. S. Mutchler (Rice), and P. J. Riley (Texas). D. L. Adams is the Project Manager. Most of the current developmental work on the cylindrical drift chamber is being carried out by participants from Houston, Rice, and Texas.

The design of the E1097 detector system is based on extensive Monte Carlo simulations performed both here and the at Rice University. The main detector system consists of a large array of scintillator bars, surrounding a cylindrical drift chamber, at the center of which is the target. The vertical angular acceptance of the detector system $\mathrm{np} \rightarrow \mathrm{pp} \pi^{-}$is approximately from $-60^{\circ}$ to $+60^{\circ}$; the horizontal acceptance (Phase II) is from $-120^{\circ}$ to $120^{\circ}$ (for Phase I the horizontal acceptance can be larger). The scintillator will provide the first level trigger, and will measure flight times (and therefore velocities) for the $\mathrm{np} \rightarrow \mathrm{pp} \pi^{-}$protons. The cylindrical chamber will be able to detect all three particles for about $75 \%$ of phase space. The chamber will measure positions perpendicular to the wires by measuring the drift time, and along the wires using charge division. This requires that the analog signal from both ends of each sense wire be read into ADCs and that some fraction of these signals be diverted into a summing circuit for discrimination and input to a TDC. It has been decided that the LeCroy FERA system will be used to provide these digitizations. Its extremely fast digitization and buf er transfer time $(20 \mu \mathrm{s})$ makes it uniquely suited to the LAMPF beam structure (120 Hz at $10 \%$ duty).

A three-body final state is characterized by 5 kinematic parameters. We will calculate 5 such parameters for each event using the directions of each of the 3 particles as determined by the drift chamber ( 6 values), and the times of flight for forward particles (normally 2 values) deduced from the scintillator array. Thus, the data set from E1097 will contain more information than is needed to reconstruct the kinematics of the events. The quality of the fit, which assumes the kinematics of $n p \rightarrow p p \pi^{-}$, is used to identify background events. It is necessary that the data be over-constrained in order to cut against background in Phase II, where the frozen spin target is expected to introduce much noise 
due to quasi-free scattering of neutrons with the bound protons in the carbon nuclei in the target. The bound events generally have large $\chi^{2}$ values than the free events due to the Fermi motion associated with their protons.

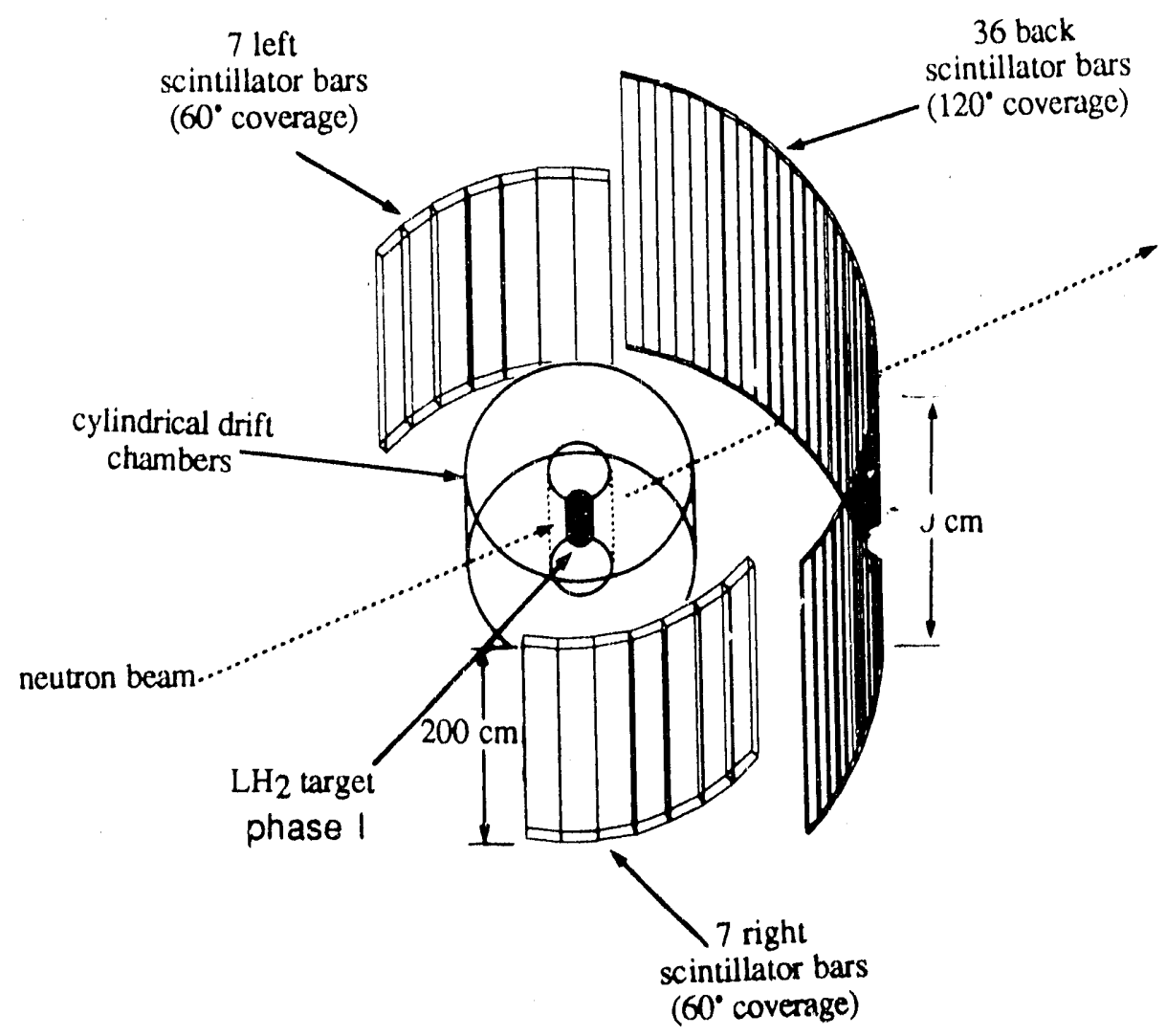

Figure 7: Schematic diagram of E1097 detector system

\section{E1097: Monte Carlo}

Initial Monte Carlo studies were performed at the University of Texas and at Rice University to test the feasibility of Phase II, in which it will be necessary to separate free events from quasifree $n(p) \rightarrow p p \pi^{-}$events where the target protons are bound in a nuclear target, such as carbon. Simulations were made with and without Fermi motion introduced in the target protons, and events reconstructed by minimizing a kinematically defined $\chi^{2}$. The studies indicate that with reasonable detector resolution, we should be able to achieve backgrounds comparable to that obtained in LAMPF experiment $815\left(\mathrm{pp} \rightarrow \mathrm{np} \pi^{+}\right)$, which involved similar separation of free from quasi free events due to the presence of a polarized target.

In order to study detector acceptances for the E1097 apparatus, we used the Monte Carlo program developed by David Adams of Rice University. This program generates kinematically correct $\mathrm{pp} \rightarrow \mathrm{pp} \pi^{-}$events, ar:d propagates the reaction products through a pre- 
defined experimental system, producing coordinates of the individual particle hits in the detectors.

From this Monte Carlo data, estimates of the geometrical acceptances for the various detectors have been determined corresponding to the percentage of reaction products ir tersecting the detector volumes. Additionally, a multiple-hit-loss ("MHL") acceptance has been calculated for the detectors, which takes into account our detectors" inability to distinguish between a single or a multiple particle hitting the same sense-wire or scintillator bar. Experimentally, this results in the apparatus detecting fewer particles per event. Applying these calculations to the four sense-wire planes (W1, W2, W3, W4 of radius $10,15,20,25 \mathrm{~cm}$, respectively) and the three scintillator arrays (S left, $S_{\text {middle, }}$ $\left.S_{\text {right }}\right)$, gives the following results for the percentage acceptance of $0,1,2$, or 3 of the reaction products:

\begin{tabular}{|c|c|c|c|c|c|c|c|c|}
\hline Detector & \multicolumn{4}{|c|}{ Geometric Acceptance (\%) } & \multicolumn{4}{|c|}{ MHL Acceptance (\%) } \\
\hline \# particles: & 0 & 1 & 2 & 3 & 0 & 1 & 2 & 3 \\
\hline W1 & 0 & $\overline{0}$ & $\overline{14}$ & 86 & 0 & 1 & 24 & $\overline{75}$ \\
\hline$\overline{\mathrm{W} 2}$ & 0 & 0 & 17 & 83 & 0 & 1 & 22 & 77 \\
\hline W3 & 0 & 1 & 21 & 78 & 0 & 1 & 25 & 74 \\
\hline W4 & 0 & 1 & 24 & 75 & 0 & 1 & 28 & 71 \\
\hline$S_{\text {left }}$ & 92 & 8 & 0 & 0 & 92 & 8 & 0 & 0 \\
\hline$S_{\text {middle }}$ & 0 & 1 & 40 & 59 & 0 & 5 & 43 & 52 \\
\hline Sright & 92 & 8 & 0 & 0 & 92 & 8 & 0 & 0 \\
\hline
\end{tabular}

Thus, the likelihood of a detector distinguishing all three reaction products is between five and ten percent lower than the geometrical acceptance value when multiple hit loss is taken into account, with the difference being proportional to the angular width of the sense wires and scintillator bars. The implications of imposing certain trigger requirements on the detectors were also investigated. For instance, if the total scintillator array is divided in half, with the requirement that at least one particle be detected by both halves, then we will lose approximately $3 \%$ of otherwise detectable events.

In order to simulate the physical effects of magnetic fields (important due to the presence of the polarized target), multiple scattering, and pion decay we will use the CERN Library simulation program GEANT. In addition to providing a more detailed description of the experiment, including realistic detector responses and tracking procedures, GEANT will be able to produce accurate graphical representations of the apparatus. Currently, we are in the process of defining our apparatus, including both dimensions and inaterial types, learning how to transform our defined objects into sensitive detectors with realistic output, and constructing a kinematics Monte Carlo code in order to simulate the reaction of interest.

The results of these Monte Carlo studies have been considered in the design of the drift chamber. For example, the need for a staggering pattern of the wire cells and the need for charge division to determine the vertical position of a track (discussed bulow) both became apparent upon analysis of the Monte Carlo data. We will continue to use the Monte Carlo in our final design of the scintillator array and its supporting structures.

\section{E1097: Drift Chamber}

The wire chamber is a $1.1 \mathrm{~m}$ long cylinder of $16.4 \mathrm{~cm}$ inner and $54 \mathrm{~cm}$ cuter diameter with 1792 high voltage (HV) wires and 448 sense wires running vertically inside. The cylindrical walls are $1 \mathrm{~mm}$ thick carbon, fabricated from epoxy-impregnated carbon fiber tape. The high voltage wires are made of gold-coated stainless steel and are 4 mils in diameter. The sense wires are 1.2 mils diameter stablohm with an internal resistance of 
100 ohms per meter. Two 1.1 meter long tubes of $1 \mathrm{~mm}$ thick carbo fiber material constitute the outer and inner sides of the cylinder. Two aluminum end caps $1^{1} / 4$ inches $(3.175 \mathrm{~cm})$ thick, $60 \mathrm{~cm}$ diameter, will hold the wires and cap the cylinder at both ends. The wires will be connected to individual preamplifiers and FERA (Fast Encoding and Readout ADC) electronics which perform rapid charge division calculations to determine the vertical position of the electron avalanche on the wire to within $1 \%$ resolution. TDCs also measure electron drift times which will give us horizontal resolution to within $0.5 \mathrm{~mm}$.

A smaller test chamber was built at the University of Texas and wired at Rice University. At Rice, Dave Adams and Tom Gaussiran tested different gas mixtures, the resolution of the sense wires, high voltage settings, the effectiveness of various cell shapes, and the suitability of FERA electronics for data acquisition. From their tests, they concluded that the resolution of the sense wires is $0.1-1 \%$ and that $\mathrm{HV}$ around $3500 \mathrm{~V}$ produces good pulses in the preamplifiers. A good gas mixture was $70 \%$ Argon, $30 \%$ Isobutane, with trace amounts of Freon. Some of the Argon will be bubbled through methanol to prevent build-1!p on the HV wires.

A major contribution of the Rice group has been the design of the electronics to carry the signals from the chamber to CAMAC digitization modules. They have designed a board to sit on the chamber which provides amplification and line drivers for 16 wires. A second board will receive the signals following 150 feet oi ribbon cable delay. This board will pick off and sum a small fraction of the signal from each end and discriminate the sum. The two analog and one discriminated outputs for each wire will then be passed along to the appropriate digitization stations. The latter board has been designed and the first prototypes are being fabricated.

The circular endplates were designed at the University of Texas Physics department and built in the UT Physics machine shop with a computer-controlled mill. They were each cut from 1.25 inch $(3.175 \mathrm{~cm})$ aluminum jigplate, $60 \mathrm{~cm}$ in diameter with $8 \mathrm{~cm}$ diameter holes in their centers. Two circular grooves, $3 \mathrm{~mm}$ wide and $4 \mathrm{~mm}$ deep, which hold the carbon fiber walls were cut $8.2 \mathrm{~cm}$ and $27 \mathrm{~cm}$ from the center of the plate.

2240 holes, each drilled and reamed to 0.126 inch $(0.320 \mathrm{~cm})$ diameter are arranged in 20 concentric rings around the center of the plate, and grouped into four groups of five rings each. The rings of sense wires have radii of 9.5 and $10.5 \mathrm{~cm}, 14.5$ and $15.5 \mathrm{~cm}$, 19.5 and $20.5 \mathrm{~cm}$, and 24.5 and $25.5 \mathrm{~cm}$ respectively. Sense wires and high voltage wires, in a ratio of $4 \mathrm{HV}$ wires to each sense wire, feed through plastic guides inserted into the holes. The holes of the rings are staggered to produce a hexagonal cell configuration. The results of the tests suggest that this cell shape will produce efficient electron avalanche on the sense wire. Furthermore, the offset pattern of the cells resolves the "left-right" ambiguity associated with drift chambers. The diagram in the following figure shows the arrangement of the HV wires and sense wires in the hexagonal cells. 


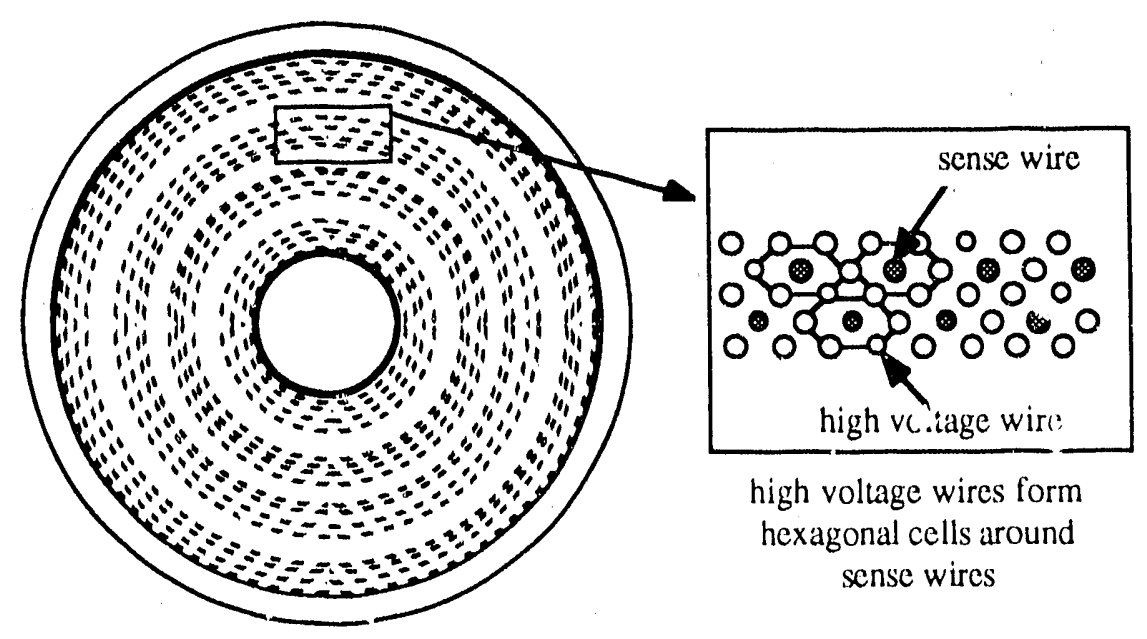

Figure 8: Endplate with hexagonal cells

For the construction of the carbon fiber walls of the chamber, a rod with "halfmoon" ribs encased in aluminum served as the mandril. The two mandrils were built at the University of Texas and consist of a $1.27 \mathrm{~m}$ long steel pipe, 2 inch $(5.08 \mathrm{~cm}) \mathrm{OD}$, with 0.25 inch $(0.635 \mathrm{~cm})$ wall thickness. There are four aluminum ribs which are made of halfmoons and flanges. This skeleton provides support for the 0.0625 inch aluminum skin rolled and then encased around it. Using this mandril as a mold, 2 mil mylar, then 1 mil aluminized kapton was over-wrapped in a continuous spiral around the aluminum skin. One mm of "prepreg" epoxy carbon fiber tape was then sf ral-wrapped around the kapton to become the wall of the final piece. A second 1 mil aluminized kapton coat was wrapped around the carbon fiber tape and taped in place at the ends of the cylinder. The entire assembly was placed in an oven and the carbon walls cured at $250^{\circ} \mathrm{F}$ overnight. After curing, the aluminum mandril and protective mylar were extracted, leaving a cylindrical shell of aluminized carbon fiber, $\sim 1 \mathrm{~mm}$ thick. The mandril was designed and constructed at the University of Texas, but the application of the mylar, kapton and prepreg was carried out at the University of Houston Streamer Chamber Facility, SCARF. At the present time one of the cylindrical walls has been fabricated, and is being tested for strength and high voltage breakdown. We are waiting the outcome of these tests before continuing with the second wall. Kathleen Johnston of the University of Houston has been instrumental in the cylinder construction and will, we hope, play an important role in the chamber assembly and installation.

As soon as the cylinder has been assembled we will begin the labor-intensive operation of stringing the wires. At the same time components will be added to the electronics boards with the goal of having the chamber constructed and a third of the electronics assembled in early 1991. The chamber will then move to Los Alamos for beam tests in the summer of 1991. 


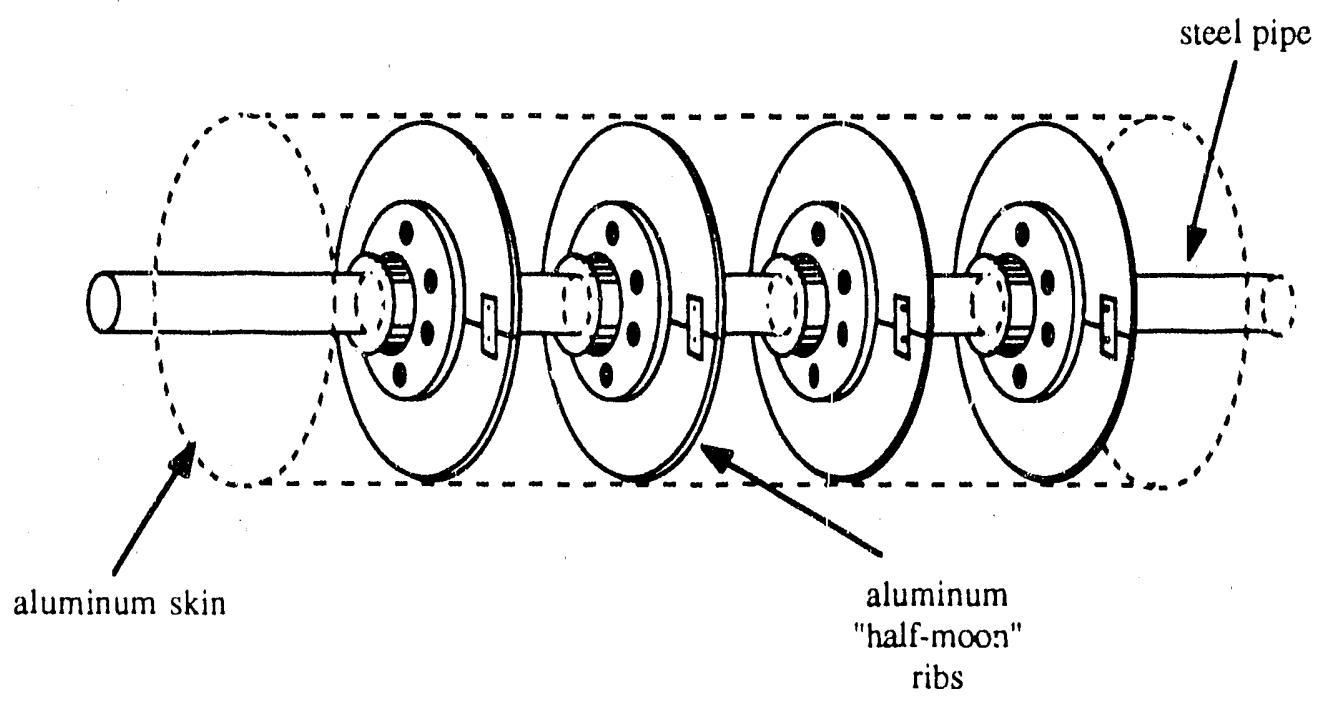

Figure 9: Construction of Mandril
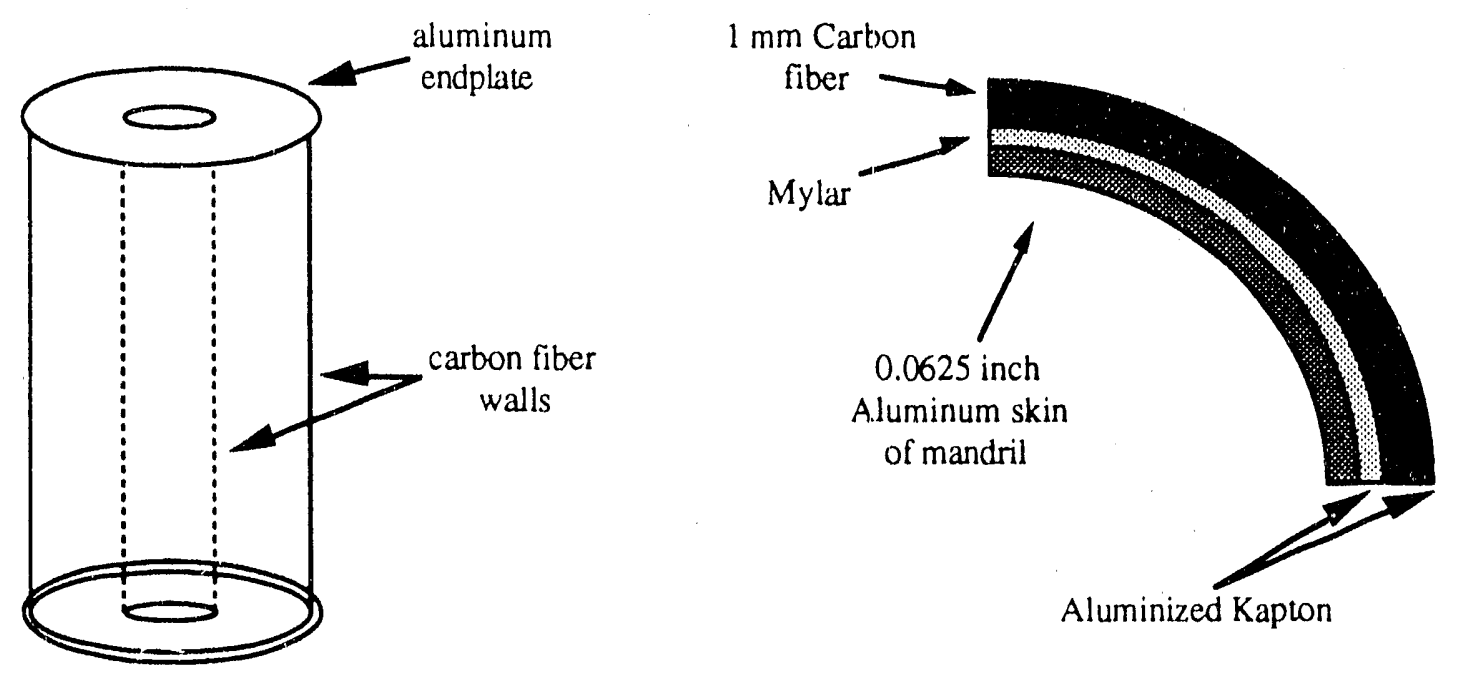

Figure 10: Basic chamber and construction of chamber wall 


\section{E. Fermilab E683, Photoproduction of High $P_{t}$ Jets in the Wide Band Beam of the Tevatron}

This experiment will study the photoproduction of high $\mathrm{p}_{\mathrm{t}}$ jets in the wide band beam of the tevatron, the highest energy photon beam available in the irorld. The QCD processes of interest are QCD Compton scattering $\gamma g \rightarrow g q$ (which dominates at high $x_{t}$ ), and quark-gluon fusion $\gamma g \rightarrow q q$. These processes are very distinctive, with the photon coupling as a point particle, giving all its energy to the two high $\mathrm{p}_{t}$ jets, and producing no beam jet.

The A-dependence of jet production from nuclei is of interest in order to study the propagation of partons through nuclear matter and to understand the space-time development of hadronization, one of the most important outstanding questions of QCD. A photon beam is a clean probe of these processes, due to the at sence of a beam jet. Also, in regions where the Compton diagram dominates, differences in propagation of quarks and gluons through nuclear matter $\mathrm{r}$ ight be observed.

Photons in the momenturn range 200 to $600 \mathrm{GeV} / \mathrm{c}$ will be tagged with a momentum uncertainty of about $2 \%$. The experimental apparatus consists of a wide-angle magnetic spectrometer, the main calorimeter array, and a forward calorimeter. The spectrometer is comprised of an SCM 1.05 inagnet with 15 planes of drift chambers and PWCs. The main calorimeter is segmented in area and depth and consists of 528 modules. An electromagnetic shower detector has oeen added to distinguish between single photons and $\pi^{\circ}$ 's. E683 shares the wide-band hall with E687.

During the last fixed target run, September-October, 1990, we took about two weeks of test beam in which several tapes were written on some of the nuclear targets (no liquid hydrogen). This yielded enough information to make us confident that the equipment and software were working correctly. We are adding two more PWC chambers in front of the existing PWC to provide better tracking in the vertical direction. The accelerator is scheduled to begin a fixed-target run at the beginning of January, 1991, at which time E683 will run for from 3 to 5 months, with at least part of the beam time being shared with E687.

Our involvement with E683 has been primarily limited to that of David Northacker, who joined UT in July, 1989 as an engineer, employed 1/2 time, but who lives and works at Fermilab. As E683 began taking shape two summers ago, the need for a gas expert became apparent, and having some experience in this area, David volunteered to oversee this part of the experiment. The basic design was approved in December 1988, and by September 1989 the system was operational. Fermilab personnel installed most of the tubing, but the racks and downstairs distribution of lines were done by David.

Since that time David's primary effort has been toward maintaining the gas system by changing bottles and repairing failed components. This includes a virtually daily observation of the system ever since the system was turned on, especially during periods where flammable gases are flowing. Although during times where beam is being taken there are frequent observations of the system made by shift personnel, at all other times David is essentially the only individual responsible for the system.

Specific details for the present include hooking up the two new MPWCs to the existing system, which should occur in the next few weeks. David anticipates continuing to maintain the gas system until the end of the experiment, and in assisting in various other projects where help is needed on an intermittent basis. 


\section{F. BNL E791: Search for $K_{L}{ }^{0} \rightarrow \mu e, K_{L}{ }^{0} \rightarrow e e$}

The present description of the fundamental particles and their electroweak and strong interactions is the Minimal Standard Model (MSM). Although this model has never been shown to be wrong, because of its many arbitrary parameters, it is suspected to be incomplete. Measurements performed in recent years make $\mathrm{i}^{+}$clear that incompleteness must originate with physics at very large mass scales. Rare decay physics probes some of these higher mass scales.

In the MSM the decay $\mathrm{K}_{\mathrm{L}}{ }^{\mathrm{O}} \rightarrow \mu \mathrm{e}$ is forbidden by conservation of electron and muon additive quantum numbers. Its observation would be the first direct evidence of physics beyond the MSM. The decays $\mathrm{K}_{\mathrm{L}}{ }^{\mathrm{O}} \rightarrow \mu \mu$ and $\mathrm{K}_{\mathrm{L}}{ }^{\mathrm{O}} \rightarrow$ ee are permitted in the MSM, but are highly suppressed. $\mathrm{K}_{\mathrm{L}}{ }^{\mathrm{O}} \rightarrow$ ee has not been seen, and observation of a branching ratio for this process that is significantly above MSlil predictions would also be evidence for new physics.

BNL EXP 791 is a high sensitivity search for $\mathrm{K}_{\mathrm{L}}{ }^{0} \rightarrow \mu e$ and $\mathrm{K}_{\mathrm{L}}{ }^{\mathrm{o}} \rightarrow$ ee. The spectrometer, located on the B5 line of the Alternating Gradient Synchrotron facility of the Brookhaven National Laboratory, is shown schematically in Fig. 11. This spectrumeter provides precise tracking with good (redundant) particle identification. Very high rate capability is achieved using custom built front-end electronics, a large parallel readout architecture, and a multi-level trigger system utilizing SLAC 3081/E emulators.

The experiment has had three production runs spanning 1988-1990. Peter Riley joined the collaboration in 1989 and participated in the 18 week 1990 run. After the 1990 production run, a beam dump or "plug" was installed in the second dipole magnet to investigate whether such a concept could be utilized for a new, factor of 10 type improvement, experiment. Based on the results of the plug test, the E791 collaboration is presently designing a new plug for additional tests to be made in early 1991 . We are involved in these designs.

The first stage (and most computer intensive) of the offline analysis of the 1990 data is now complete. This analysis involved processing all data tapes using event reconstruction algorithms. From this analysis we can already give a preliminary sensitivity of the 1990 data set for a single $\mathrm{K}_{\mathrm{L}}{ }^{\mathrm{O}} \rightarrow \mu \mathrm{e}$ event. The single event sensitivity is about $3 \mathrm{X}$ $10^{-11}$. When all three data sets are combined it is expected that this single event sensitivity will be about $1.5 \times 10^{-11}$ (implying a final limit $\leq 10^{-11}$ at the $90 \%$ confidence level. )

In addition to providing the world's best limit on $\mathrm{K}_{\mathrm{L}}{ }^{\mathrm{O}} \rightarrow \mu \mathrm{e}$, the experiment has accumulated a large number of $\mathrm{K}_{\mathrm{L}} \mathrm{O} \rightarrow \mu \mu$ events. It is anticipated that the combined 19881990 runs will give a total number of about 600 events ( 350 from the 1990 run). This will imply knowledge of the branching ratio to about $5 \%$. 


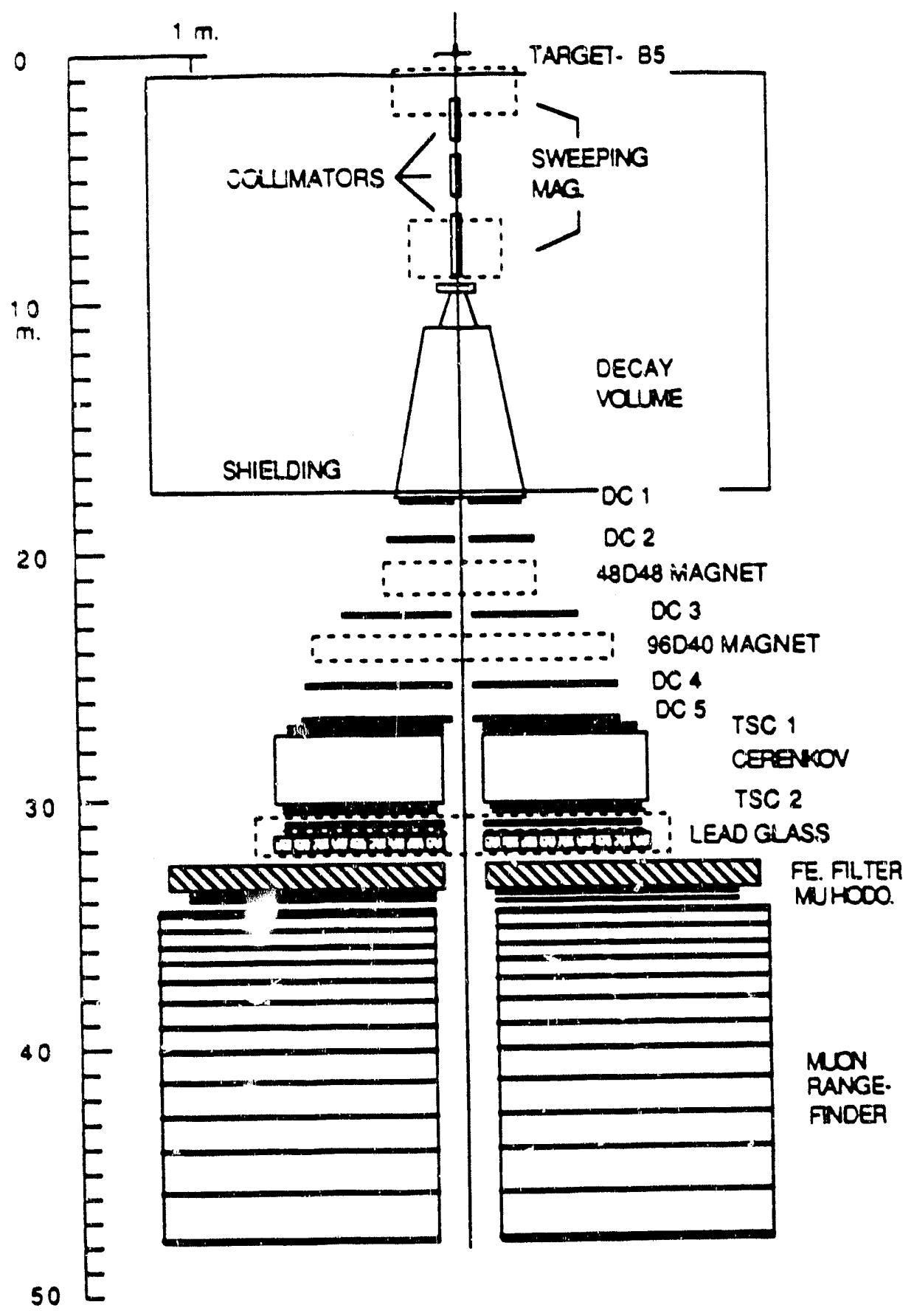

Figure 11: Schematic drawing of the kaon beam line and detection apparatus for BNL EXP 791. 


\section{Status of Experiments}

LAMPF E1072: pp Elastic Absolute Cross Section Measurements Spokesperson: M. W. McNaughton Participating Instimutions: LANL, University of Texas, Texas A\&M University, Rutgers University, UCLA

Status: Running period June-September, 1988. Data analysis is in progress.

Comments: The aim of this experiment, which was carried out during the summer of 1988 , is to measure the differential cross section for pp elastic scattering to an absolute accuracy of $1 \%$. The data were taken at 500-,580-, 650-, 733-, and $800-\mathrm{MeV}$ beam energies and over the angular range $15^{\circ}$ to $90^{\circ} \mathrm{c} . \mathrm{m}$. The data are being analyzed by a joint effort from the University of Texas (Kok Heong McNaughron) and Texas A\&M University.

TRIUMF E372: Single Pion Production in np Scattering Spokesperson: N. E. Davison Participating Institutions: University of Manitoba, University of Alberta, California State University, TRIUMF, Rice University, University of Houston, University of Texas.

Status: Approved. Test runs were successfully carried out during 1988. A 10-day run with polarized beam was successfully carried out in the period Sept. 12 - Sept. 22, 1990. An additional one-week run period with polarized beam is scheduled for Nov. 21-27, 1990. This should complete the E372 data taking. Data are currently being analyzed at the University of Manitoba and at the University of Texas.

LAMPF E876: Spin Transfer Measurements for np Elastic Scattering Spokesperson: M. W. McNaughtori Participating Institutions: LANL, University of Texas, UCLA Status: Approved with A priority for 1500 hours of L-type beam between 650 and 800 $\mathrm{MeV}$ in the summer of 1990 . Requires the high intensity optically pumped $\mathrm{p}^{-}$ion source. The experiment ran smoothly with few interruptions through all of June, 1990, using a 15liter liquid hydrogen $\left(\mathrm{LH}_{2}\right)$, the largest ever used at LAMPF. On July 4 an accident occured which destroyed the 15-liter target, and damaged several of the detectors. By September, data taking was resumed using a 3-liter target. This target was replaced with the rebuilt 15-liter target in early Octoler, and data taking continued until the end of the cycle. Final E876 runs should be completed during the spring and summer of 1991.

FERMILAB E683: Photoproduction of high $\mathrm{p}_{\mathrm{t}}$ jets in the wide band beam of the Tevatron

Spokesperson: M. D. Corcoran

Participating Institutions: Rice University, University of Texas, Lehigh University, Ball State University, University of Wisconsin, FERMILAB

Stalus: David Northacker is working approximately one-half time on this experiment. During September-October we took about two weeks of test beam which served to check the status of both hardware and software. The accelerator is scheduled to begin a fixedtarget run at the beginning of January, 1991, at which time E791 will run from 3 to 5 months, with a least part of the beam time being shared with $E 687$.

BNL E791: Study of Very Rare $K_{L}{ }^{\circ}$ Decays

Spokespeople: W. R. Molzon and R. D. Cousins

Participaring Institutions: University of California, Irvine; University of California, Los Angeles; Los Alamos National Laboratory; Stanford University; Temple University; University of Texas; College of William and Mary 
Status: The 1990 run time, the last one planned for E791, was an 18 week period from January-May. (Previous runs were made in 1988 and 1989 respectively, before our involvement in the experiment.)

Comments: The main goal of E791 is to search with high sensitivity for th: separate lepton number non-conserving decay $\mathrm{K}_{\mathrm{L}}{ }^{\mathrm{O}} \rightarrow \mu \mathrm{e}$. The first stage of the offline analysis of the 1990 data is now complete. Wisn all three data sets (runs from 1988, 1989, and 1990) are combined, it is expected that the single event sensitivity for $\mathrm{K}_{\mathrm{L}}{ }^{\mathrm{O}} \rightarrow \mu \mathrm{e}$ will be about $1.5 \mathrm{X}$ $10^{-11}$, which is expected to be the world's best limit for this decay.

LAMPF E1097: Single Pion Production in np Scattering.

Spokespeople: D. L. Adams, N. E. Davison, G. S. Mutchler, P. J. Riley. Project Manager, David Adams.

Participating Institutions: ANL, UCLA, California State University, University of Houston, University of British Columbia, LANL, University of Manitoba, University of Alberta, Queen Mary College, Rice University, University of Texas, Texas A \& M University.

Status: Approved with $A^{-}$priority by the LAMPF PAC, and by LAMPF management. Testing of the cylindrical drift chamber is scheduled for 1991. Phase I (unpolarized $\mathrm{LH}_{2}$ ) target, is scheduled for 1992; Phase II (Polarized, frozen spin hydrogen target) is scheduled for 1993.

Comments: This experiment will measure the differential cross section, and the spin observables $A_{N O}, A_{L O}, A_{S O}, A_{S L}, A_{N L}$, and $A_{L L}$ for the single pion production reaction $\mathrm{np} \rightarrow \mathrm{pp} \pi^{-}$at neutron beam energies in the range $500-800 \mathrm{MeV}$. With the polarized target used in Phase II of E 1097 a three charged-particle trigger will be required in order to enable us to discriminate against the quasifree $n p \rightarrow p p \pi^{-}$background events. For this, a detector system with a much larger acceptance than is available in TRIUMF E372 is needed. Because of the LAMPF low duty factor (especially for energies other than $800 \mathrm{Mev}$ ), it is essential that we be able to accept more than one event per macropulse. E1097 therefore requires a large acceptance detector sysiem, new (cylindrical) drift chambers, and fast electronics. We have identified tasks, designated responsibilities, and established a schedule for E1097 milestones. We have built a test drift chamber which has provided us information on the design and performance of the cylindrical drift chambers, and on the electronics needed for them. The construction of the drift chamber is well in hand, and should be completed by early 1991. Most of our current E1097 efforts are being carried out by collaborators from the University of Texas, Rice University, and the University of Houston. 
Riley - DOE 1990: Page 28

\section{Personnel Working on the Project}

Peter J. Riley, Professor of Physics

Kok Heong McNaughton, Computer Programmer (3/4 time)

David Northacker, Engineer (1/2 time, starting July, 1989)

Graduate Students:

Mark Bachman (1/2 time)

David Ambrose (1/4 time, starting July, 1989)

John Johnson (1/2 time, starting September, 1989)

Undergraduate Students:

Dan Allcock (June-August, 1990)

Patrick Coffey (1/4 time, starting Sept. 1990)

Peter Riley has participated in E1072, E372, E876, and E791 data runs, and has been much involved in the planning for E1097. Much of his time is now being spent on E1097 cylindrical drift chamber development. Kok Heong McNaughton lives and works in Los Alamos, and is responsible for the software for all of our experiments there. In addition, she assists in data acquisition runs, and in data analysis at LAMPF. David Northacker lives and works at Fermilab, and assists in E683. Mark Bachman, whose Ph. D. dissertation research is based on TRIUMF E372, has been spending most of his time at the University of Texas on software development for E372. At present he is working to analyze E372 data. The Ph. D. thesis topic of David Ambrose is expected to be E1097. David spent the past summer at LAMPF, helping in E876 data acquisition, and has returned to the University of Texas for the academic year to complete his course requirements. Johin Johnson has completed his $\mathrm{Ph}$. D. course requirements, and is residing in Los Alamos, where he is working on E876. Dan Allcock participated in E876 runs during the summer of 1990 , and assisted in developing the E372 diagnostic software during the summer of 1989. Pat Coffey has worked with GEANT development and preliminary benchmark tests of the IBM RISC machine (he has previous experience with UNIX). He has also begun design studies for the E1097 scintillator support structures. 
Riley - DOE 199(): Page 29

\section{PUBLICATIONS and PRESENTATIONS}

\section{PUBLICATIONS}

"Some Proton Spin Observables Obtained in p-d Elastic Scattering at 500 and $800 \mathrm{MeV}$," Physics Letters B 194, 338, (1987), A. Rahbar, B. Aas, E. Bleszynski, M. Bleszynski, G. Ganezer, G. J. Igo, F. Irom, B. E. Bonner, O. van Dyck, M. W. McNaughton, J. B. Roberts, C. L. Hollas, R. D. Ransome, and P. J. Riley.

"Polarization Cbservables for the Reaction $\mathrm{pp} \rightarrow \mathrm{pp} \pi^{\mathrm{O}}$ at $800 \mathrm{MeV}$," Physics Letters B 197, 23, (1987), P. J. Riley, M. Bachman, C. L. Hollas, K. H. McNaughton, Shen-wu Xu, B. E. Bonner, O. B. van Dyck, J. McGill, M. W. McNaughton, J. C. Peng, R. R. Silbar, J. Dubach, and W. M. Kloet.

"Evidence Against Broad Dibaryons," Physical Review Letters 60, 901, (1988), R. L. Shypit, D. V. Bugg, D. M. Lee, M. W. McNaughton, R. R. Silbar, N. M. Stewart, A. S. Clough, C. L. Hollas, K. H. McNaughton, P. J. Riley, and C. A. Davis.

Comment on "Evidence Against Broad Dibaryons," Reply, Physical Review Letters 61, 2385, (1988), R. L. Shypit, D. V. Bugg, D. M. Lee, M. W. McNaughton, R. R. Silbar, N. M. Stewart, A. S. Clough, C. L. Hollas, K. H. McNaughton, P. J. Riley, and C. A. Davis.

"ASL, ALL, and $A_{N O}$ for $N N \rightarrow \pi d$ at 492, 729, and $796 \mathrm{MeV}, " N u c l e a r$ Physics A477, 541, (1988), R. L. Shypit, D. V. Bugg, D. M. Lee, M. W. McNaughton, R. R. Silbar, N. M. Stewart, A. S. Clough, C. L. Hollas, K. H. McNaughton, P. J. Riley, and C. A. Davis.

"Neutron-Proton Analyzing Power Measurements from 375 to $775 \mathrm{MeV}$," C. R. Newsom, C. L. Hollas, R. D. Ransome, P. J. Riley, B. E. Bonner, J. G. Boissevain, J. J. Jarmer, M. W. McNaughton, J. E. Simmons, T. S. Bhatia, G. Glass, J. C. Hiebert, L. C. Northcliffe, W. B. Tippens, Physical Review C39, 965, (1989).

"Spin Correlation Parameter $A_{N N}\left(\theta^{*}\right)$ for $n \cdot p$ Elastic Scattering at $790 \mathrm{MeV}, "$ S. Nath, G. Glass, J. C. Hiebert, J. A. Holt, R. A. Kenefick, L. C. Northcliffe, D. P. Grosnick, D. Lopiano, Y. Ohashi, T. Shima, H. M. Spinka, R. Stanek, T. S. Bhatia, J. J. Jarmer, P. J. Riley, S. Sen, J. A. Faucett, G. Kyle, R. H. Jeppesen, G. E. Tripard, Physical Review D39, 3520, (1989).

"NN $\rightarrow N N \pi$ at LAMPF and TRIUMF," P. J. Riley, in American Institute of Physics Conference Proceedings No. 187, Vol. 1, pages 613-622, High-Energy Spin Physics, Eighth International Symposium, Editor; Kenneth Heller, New York, 1989.

"Spin Physics at LAMPF," Peter J. Riley, in Spin and polarization Dynamics in Nuclear and Particle Physics, Trieste, Italy, January, 1988. Edited by A. O. Barut, Y. ONel, A. Penzo. Published by World Scientific, 1990, pages 241-260.

"Spin Dependence of pp $\rightarrow n p \pi^{+}$from 492 to $796 \mathrm{MeV}$," R. L. Shypit, D. V. Bugg, A. H. Sanjari, D. M. Lee, M. W. McNaughton, R. R. Silbar, C. L. Hollas, K. H. McNaughton, P. Riley, C. A. Davis., Physical Review C40, 2203, (1989).

"3-Spin Measurements in pp $\rightarrow$ pp at $730 \mathrm{MeV}$," M. W. McNaughton, S. Penttila, K. H. McNaughton, P. J. Riley, D. L. Adams, J. Bystricky, E. Gulmez, and A. G. Ling, Physical Review C41, 2809, (1990). 
"Analyzing Power Measurements in $\mathrm{pn} \rightarrow \pi^{-} \mathrm{pp}\left({ }^{1} \mathrm{~S}_{\mathrm{O}}\right)$," Mark Bachman, Peter Riley, and Chalrles Hollas, Physical Review C, (Brief Reports), October, 1990.

"Accurate Intensity Measurements for Proton Beams with a $201 \mathrm{MHz}$ Structure," E. Gulmez, A. G. Ling, C. A. Whitten, Jr., J. F. Amann, M. W. McNaughton, T. Noro, D. L. Adams, V. R. Cupps, R. D. Ransome, G. Glass, A. J. Simon, K. H. McNaughton, and P. J. Riley, to be published in Nuclear Instruments and Methods $A$.

"Absolute Differential Cross Section Measurements for Proton-Deuteron Elastic Scattering at 641.3 and $792.7 \mathrm{MeV}$," E. Gulmez, S. Beedoe, A. G. Ling, C. A. Whitten, Jr., M. W. McNaughton, J. R. Santana, D. L. Adams, V. R. Cupps, A. J. Simon, M. L. Barlett, K. H. McNaughton, and P. J. Riley, submitted for publication in Physical Review $C$.

\section{ABSTRACTS}

"Measurements of Two- and Three-Spin Parameters in p-d Elastic Scattering at $8(0) \mathrm{MeV}$," M. Begala, G. Kahrimanis, K. McNaughton, P. Riley, D. Wolf, D. Adams, S. Bedoe, G. Bystricky, E. Gulmez, G. Igo, A. Ling, M. Nasser, G. Glass, and M. McNaughton, Bulletin of the American Physical Society 33, 1472, (1988),

"The Absolute P-D Elastic Scattering cross Sections at 750 and $800 \mathrm{MeV}$," E. Gulmez, S. Bedoe, A. G. Ling, C. A .Whitten, Jr., M. W. McNaughton, J. R. Santana, M. L Barlett, K. H. McNaughton, P. J. Riley, V. R. Cupps, D. L. Adams, A. J. Simon, Bulletin of the American Physical Society 34, 1140, (1989).

"Accurate Intensity Measurements for Proton Beams with a $200 \mathrm{Mhz}$ Structure," E. Gulmez, A. G. Ling, C. A.. Whitten, Jr., J. F. Amann, M. W. McNaughton, T. Noro, K. H. McNaughton, P. J. Riley, V. R. Cupps, R. D. Ransome, D. L. Adams, G. Glass, A. J. Simon, Bulletin of the American Physical Society 34, 1798, (1989).

\section{PRESENTATIONS}

"Spin Physics at LAMPF," Peter Riley, Invited Talk, 8th International Adriatico Research Conference on Spin and Polarization Dynamics in Nuclear and Particle Physics, January, 1988, Tiieste, Italy.

"NN $\rightarrow$ NN $\pi$ at LAMPF and TRIUMF," Peter Riley, Invited Talk, $8^{\text {th }}$ International Symposium on High Energy Spin Physics, September 12-17, 1988, University of Minnesota.

"Spin Physics at Medium Energies," Peter Riley, University of Texas Medium/High Energy' Physics seminar, February 16, 1989.

"Spin Physics at LAMPF," Peter Riley, Invited Talk, Western Regional Nuclear and Particle Physics Conference, Lake Louise, Alberta, Canada, February 17-19, 1989.

"The LAMPF Long Range Plan," Peter Riley, Invited Talk, Division of Nuclear Physics Town Meeting: Light Hadronic Probes, May 22-23, 1989, Santa Fe, New Mexico.

"The LAMPF Long Range Plan," Peter Riley, Invited talk, TRIUMF KAON Factory Users Workshop, Vancouver, B. C. Canada, July 10-11, 1989. 
Riley - DOE 1990: Page 31

Ph. D. Dissertation

Wolfenstein Polarization Observables for the Reactions $p p \rightarrow n p \pi^{+}$and $p p \rightarrow p p \pi^{\circ}$ at 650 and $800 \mathrm{MeV}$, Shen-Wu Xu, Ph. D. Thesis, University of Texas at Austin, June, 1988. 
VI. Title Pages of Publications

Copyrighted Wbatiaets Lamod 
